Draft Version July 27, 2015

Preprint typeset using $\mathrm{LAT}_{\mathrm{E}} \mathrm{X}$ style emulateapj v. 5/2/11

\title{
HST ASTROMETRY IN THE 30 DORADUS REGION: MEASURING PROPER MOTIONS OF INDIVIDUAL STARS IN THE LARGE MAGELLANIC CLOUD
}

\author{
Imants Platais ${ }^{1}$, Roeland P. van der Marel ${ }^{2}$, Daniel J. Lennon ${ }^{3}$, Jay Anderson $^{2}$, Andrea Bellini $^{2}$, Elena Sabbi $^{2}$, \\ Hugues SAnA ${ }^{2}$, Luigi R. Bedin ${ }^{4}$ \\ Draft version July 27, 2015
}

\begin{abstract}
We present measurements of positions and relative proper motions in the 30 Doradus region of the Large Magellanic Cloud (LMC). We detail the construction of a single-epoch astrometric reference frame, based on specially-designed observations obtained with the two main imaging instruments ACS/WFC and WFC3/UVIS onboard the Hubble Space Telescope (HST). Internal comparisons indicate a sub milli-arc-second (mas) precision in the positions and the presence of semi-periodic systematics with a mean amplitude of $\sim 0.8$ mas. We combined these observations with numerous archival images taken with WFPC2 and spanning 17 years. The precision of the resulting proper motions for well-measured stars around the massive cluster R 136 can be as good as $\sim 20 \mu \mathrm{as} \mathrm{yr}^{-1}$, although the true accuracy of proper motions is generally lower due to the residual systematic errors. The observed proper-motion dispersion for our highest-quality measurements is $\sim 0.1 \mathrm{mas} \mathrm{yr}^{-1}$. Our catalog of positions and proper motions contains 86,590 stars down to $V \sim 25$ and over a total area of $\sim 70$ square arcmin. We examined the proper motions of 105 relatively bright stars and identified a total of 6 candidate runaway stars. We are able to tentatively confirm the runaway status of star VFTS 285, consistent with the findings from line-of-sight velocities, and to show that this star has likely been ejected from R 136. This study demonstrates that with HST it is now possible to reliably measure proper motions of individual stars in the nearest dwarf galaxies such as the LMC.
\end{abstract}

Subject headings: astrometry - galaxies: Magellanic Clouds: individual (30 Dor)

\section{INTRODUCTION}

A number of proper-motion measurements are available for the Small and Large Magellanic Clouds - the Milky Way's nearest irregular dwarf galaxies - which address their bulk absolute proper motion and rotation (e.g. Kallivavalil et al. 2013; Vieira et al. 2010; van der Marel \& Kallivayalil 2014). The accuracy of relative proper motions for individual stars with HST has been steadily increasing and now is approaching the $\sim 0.05$ mas $\mathrm{yr}^{-1}$ level, demonstrated for a sample of Galactic globular clusters (Bellini et al. 2014). This opens up an opportunity to measure reliable individual proper motions for fast-moving stars in the LMC. One such class of objects in the Magellanic Clouds are massive OB stars, some of which are thought to move at velocities close or exceeding $100 \mathrm{~km} \mathrm{~s}^{-1}$ and, hence, should be detectable from HST observations spanning two decades. These short-lived massive luminous stars play a major role in galaxy evolution by affecting the interstellar medium via chemical enrichment and other processes. One of the best sites for detailed studies of such stars is the 30 Doradus (hereafter - 30 Dor) region of the LMC, which harbors a rich population of O-type stars,

\footnotetext{
imants@pha.jhu.edu

${ }^{1}$ Department of Physics and Astronomy, Johns Hopkins University, 3400 North Charles Street, Baltimore, MD 21218, USA

2 Space Telescope Science Institute, 3700 San Martin Drive, Baltimore, MD 21218, USA

3 European Space Astronomy Centre, Camino bajo del Castillo, Urbanizacion Villafranca del Castillo, Villanueva de la Cañada, 28692, Madrid, Spain

${ }^{4}$ INAF-Osservatorio Astronomico di Padova, Vicolo dell'Osservatorio 5, I-35122 Padova, Italy
}

both in the form of star clusters and isolated objects (Walborn \& Blades 1997). The existence of the latter is puzzling because it contradicts the governing paradigm that stars are born in clusters. An additional poorlyunderstood feature of O-type stars is the very high frequency of binarity and higher-order multiplicity among these stars (Mason et al. 2009; Sana et al. 2012). The 30 Dor region is unique in the sense that it is the nearest extragalactic site where such stars are concentrated and yet are bright enough to permit high-resolution spectroscopy with ground-based facilities. Taking advantage of this favorable situation, the VLT-FLAMES Tarantula Survey (VFTS; Evans et al. 2011) has obtained multiepoch spectroscopy of over 800 massive OB stars. A series of papers (c.f., Sana 2013) based upon these observations have addressed such questions as: isolated high-mass-star formation, internal-velocity dispersion of a massive cluster, rotational characteristics of OB stars, the fraction of binaries and triples among OB stars.

One of the most interesting phenomena among OBtype stars is the presence of runaway stars (stars with large peculiar velocities relative to their general velocity distribution). The space frequency of such stars in the Milky Way has been studied by Stone (1991), showing that more than $\sim 40 \%$ of O-type stars could be runaways, while for the B-type stars this fraction drops tenfold. Two basic scenarios have been proposed to explain the presence of OB runaways. Assuming that massive stars are born in star clusters and knowing that more than half of Galactic O-type stars are binaries (Sana et al. 2012; Chini et al. 2012), it is thought that, if one component of a binary explodes as a core-collapse supernova, then the remaining star may attain a large kick veloc- 
ity and, hence, be ejected from a cluster (e.g., Blaauw 1961; Hoogerwerf et al. 2000). The second scenario assumes that a high-mass star is likely to be ejected from a cluster via the dynamical mechanism of 3-body encounters (e.g., Gvaramadze \& Gualandris 2011). If a massive young star is found in isolation, then there are two possibilities: it could be a runaway and in such a case it should have a parent star cluster not too far away, or it could have been formed in isolation and would show no kinematic signature of a runaway. This can be tested by measuring the line-of-sight (LOS) velocities and proper motions. The latter can be translated into velocities, if the distance is known with a reasonable accuracy. At the distance of the LMC the depth effect is negligible, thus making it ideal for such measurements. Furthermore, proper motions have a compelling advantage over line-of-sight velocities since they provide the direction of motion in addition to its amplitude. In other words, they can constrain the probable site of origin of a runaway star.

The 30 Doradus region of the LMC is an ideal environment to test models for runaway stars as it has the highest density of massive stars in the LMC and includes a number of apparently isolated very massive stars, e.g., the confirmed runaway star VFTS 016 (Evans et al. 2010). Indeed VFTS 016 is a prime candidate for the dynamical ejection mechanism due to its extreme youth and high mass, implying that the possible origin for this object is Radcliffe 136 (R 136) cluster itself. However, this cluster is too young $(\sim 2 \mathrm{Myr})$ to have yet had a supernova explosion. Only accurate proper motions can constrain the transverse velocity of VFTS 016 and, thus, test the assumption that it is a former member of $\mathrm{R} 136$.

\section{HST OBSERVATIONS OF 30 DOR}

We started an HST astrometric survey of the 30 Dor region (GO-12499; PI: D. Lennon), designed using extensive prior knowledge about the massive stars in this area. This project is part of the HSTPROMO collaboration ${ }^{5}$ - a set of $H S T$ projects aimed at improving our understanding of the dynamics of stars, star clusters, and galaxies in the nearby Universe through measurements of proper motions and their interpretation (e.g., van der Marel et al. 2014). The HSTPROMO collaboration draws on techniques, tools, and expertise developed over two decades of astrometric research with various $H S T$ imaging instruments.

The ground-based VFTS provides multi-epoch optical spectroscopy of $\sim 800$ OB stars in the Large Magellanic Cloud (Evans et al. 2011). This spectroscopic survey is centered around the massive and young star cluster R 136 and it extends outwards up to $r \sim 10^{\prime}$. Our matching $H S T$ proper-motion survey was optimized to cover one quadrant of the VFTS field-of-view (FOV), the entire extent of the R 136 cluster and its adjacent structures of high stellar density, and to include a bona fide runaway star VFTS 016. Figure 1 shows the spatial coverage of our proper-motion survey with respect to the distribution of VFTS targets. The crosshairs indicate the location of the most massive Wolf-Rayet star R 136a1, usually adopted as a center of the entire cluster R 136

5 For details see the HSTPROMO homepage at http://www.stsci.edu/ $\sim$ marel/hstpromo.html
(Evans et al. 2011; Hénault-Brunet et al. 2012).

Our new HST observations of 30 Dor were obtained with two imaging instruments: the Wide Field Camera 3 (WFC3) and its UVIS channel - sensitive at ultraviolet and optical wavelengths - and the Advanced Camera for Surveys (ACS) through its Wide Field Channel (WFC). In order to maximize the field coverage, both instruments observed in parallel, with the WFC3/UVIS designated as the primary camera. The FOV of WFC3/UVIS covers $\sim 7$ square arcmin of the sky, while the ACS/WFC covers $\sim 11$ square arcmin. The angular separation between the centers of these two cameras in the focal-plane is $\sim 6^{\prime}$. Such a configuration requires a minimum of two pointings to achieve a contiguous, quilt-patterned field coverage by both cameras. Formally, the entire VFTS field can be covered by $\sim 17$ appropriately-spaced pointings, but that would result in apparent "holes" in coverage and very little overlap between any two adjacent images (frames). For the sake of high-precision astrometry with $H S T$, it is advantageous to have a $\sim 50 \%$ overlap between adjoining frames, thus providing a reasonable compromise between the minimum number of needed pointings and the desire to image the largest possible contiguous area of the sky. In the case of the 30 Dor area, this amounted to 15 pointings and $~ 80 \%$ of VFTS targets covered, as shown by Figs. 12, Thus, the entire astrometric field in its global metachip coordinates is approximately $13^{\prime} \times 16^{\prime}$ in extent, rotated by $\sim 35^{\circ}$ from the East to North.

In Figure 2, a semi-rectangular pointing pattern is generated using the sky location of the primary WFC3/UVIS frames. Each of the 15 blue squares represents a separate pointing. Each adjacent pointing is half-a-frame apart in the long direction of a field and it has a $\sim 22 \%$ overlap in the short direction. Each pointing is marked by the visit number (set) in our $H S T$ program and corresponds to one complete orbit. Sets 04, 05, and 53 are rotated by $\sim 10^{\circ}$ with respect to the bulk of the sets. This rotation was necessary in order to have an appropriate pair of guide stars. The parallel observations with ACS/WFC (red squares) produced a different pattern of overlaps, where the largest overlap reaches $\sim 43 \%$ only.

To enhance the sampling of the point-spread function (PSF) and have a better handle on short-scale astrometric distortions, we obtained multiple dithered exposures at each pointing, as shown in Fig. 3. We used equalsize consecutive steps of $10^{\prime \prime}$ along both pixel axes of WFC3/UVIS. In this figure, the sub-pointing $B$ defines our base pointing for Set 01 shown in Fig. 2. At the sub-pointing 1, one short and one long exposure were obtained. The remaining sub-pointings were used to obtain a single long exposure only. The applied dither pattern for WFC3/UVIS resulted in corresponding shifts almost entirely along the $y$ pixel axis for ACS/WFC. The short exposures amounted to $35 \mathrm{~s}$ for WFC3/UVIS and $32 \mathrm{~s}$ for ACS/WFC. For WFC3/UVIS, the long exposure was either 699 or $507 \mathrm{~s}$, while for WFC3/UVIS, it was either 640 or 377 s. The observations of 30 Dor were made on 2011 October 3-8, with the Set 53 obtained on Oct 29, 2011. While the timing sequence follows the order of observational sets, one set (04) was taken right after set 16.

All observations were obtained with the F775W filter (similar to the Sloan Digital Sky Survey's $i$ filter), which 
was considered the best choice for astrometric purposes based on our prior experience. We also chose F775W because it provides the best chance of measuring accurate positions for the brightest stars, which are saturated even in the short exposures. A number of temporallystable features ("bumps") near the core of a saturated PSF are best defined in this filter, thus providing an opportunity to use them for astrometry. Additional details about the mapping of the 30 Dor region are described in Sabbi et al. (2013). That paper also provides an image mosaic of the field and preliminary photometric properties of identified sources. Here, we focus on the initial analysis of proper motions based upon comparison to the existing HST archival data. As part of GO-12499, we have recently obtained a succesful additional epoch of astrometric HST observations which will serve to derive proper motions over the entire FOV indicated in Fig. 2. These observations and a new set of complete proper motions will be discussed in a future paper.

\subsection{Archival HST observations of 30 Dor}

The richest collection of archival HST images for the 30 Dor region has been provided by the now-retired Wide Field Planetary Camera 2 (WFPC2). We selected and examined a total of 223 frames obtained in the course of 13 General Observer (GO) programs during 1994-2003. Table 1 provides basic characteristics of these frames, such as the GO Program ID, the total number of frames, the filter selection, the mean epoch of observations, and the target coordinates (in time: hr, min, sec for Right Ascension and in angular units: deg, min, sec for Declination). A program is split up if the target coordinates differ significantly, that is more than by a half of the PC1's angular size (the smallest among the four WFPC2 detectors). For GO 8059 (epoch=2000.25), there are numerous frames with a slightly varying pointing, hence a range of coordinates is indicated. There is a large variety of exposure times, ranging from 3 to $1400 \mathrm{~s}$. This enables us to cover the entire magnitude range of our target stars, albeit unevenly across the FOV. The spatial distribution of the WFPC2 observations is dominated by a large number of pointings on the rich star cluster R 136 . The remaining pointings are random, mainly dictated by the HST configuration at the time of executed pureparallel observations, such as for GO 8059. We note that the largest time baselines with respect to our new data reach 17 years, allowing determination of proper motions with high precision.

\section{DATA REDUCTION}

In this section, we present a detailed description of all the steps required to proceed from flat-fielded frames with three $H S T$ imaging instruments to constructing astrometric catalogs in the system of a geometricallycorrect reference frame and, finally, to calculating proper motions. At the beginning of this project, we adopted the initial source list from Sabbi et al. (2013) with over 87,000 stars brighter than the instrumental magnitude 6

\footnotetext{
${ }^{6}$ Instrumental magnitude is defined as $-2.5 \log \Sigma(\mathrm{DN} \times$ Gain $)$ where DN is the number of "electrons" counted under the best-fit ePSF within the inner $5 \times 5$ pixels and the resulting sum is then scaled up to represent the total flux within the radius of 10 pixels. Note that the ACS/WFC and WFC3/UVIS CCD detectors have
}

of $-9.3 \mathrm{mag}$ (corresponding to $\mathrm{S} / \mathrm{N} \sim 72$ ), detected in all available long-exposure frames. The positions of these stars are in the system of WFC3/UVIS pixels, mosaiced into a master list as described in Sect. 4.1 of Sabbi et al. (2013). The resulting mosaic is $\sim 26,000 \times 20,000$ pixels large. The first step for us was to start with this master list and then construct a reference frame (catalog) with higher astrometric accuracy. Our approach provides estimates of internal precision at various stages of building an astrometric catalog.

\subsection{Image centroids and geometric distortion}

The recent epoch GO-12499 science frames are available in various output formats from the STScI standard data reduction pipeline. We used the bias-subtracted, dark-subtracted, flat-fielded, and charge-transfer inefficiency (CTI) corrected frames, encoded as flc.fits files. All WFC3/UVIS and ACS/WFC frames were converted into a metachip format with all real flux data translated into the space-saving integers. The empirical pixel-based correction for CTI (e.g., Anderson \& Bedin 2010) in the WFC3/UVIS data was run as a stand-alone option, but this software is expected to be integrated into the STScI pipeline soon.

In order to calculate the centroids and fluxes for all detections in ACS/WFC images, we used the publicly available software code img2xym_WFC.09x10 (Anderson \& King 2006), modified to apply the necessary corrections for geometric distortion in situ. This code employs empirical, spatially-variable point-spread function (ePSF) to measure precise position and instrumental magnitude for each of the detections (star, extragalactic object, or artifact). The ending "09×10" indicates that there are $9 \times 5$ fiducial ePSFs for each of the ACS/WFC chips. Because our observations were made after the Servicing Mission 4, which restored the functionality of ACS/WFC camera, it was decided to obtain new ePSFs for the filter F775W based entirely on the observations of 30 Dor. A combination of small and large dithers among the images was instrumental in constructing reliable pixel-phase-bias-free average ePSFs detailed in Anderson \& King (2006). We also made improvements in the corrections for geometric distortion in this filter by updating a look-up table and accounting for time-dependent variations in the linear terms (Anderson 2007; Ubeda et al. 2013).

For WFC3/UVIS, a sparser array of $7 \times 8$ fiducial ePSFs was adopted because the FOV of this camera is smaller and spatial variations of PSF are less pronounced than those for ACS/WFC. The WFC3/UVIS library ePSFs were constructed from the observations of globular cluster $\omega$ Centauri taken shortly after the installation of this camera (Anderson, in preparation). To correct for geometric distortion in the positions obtained from observations with WFC3/UVIS, we used the high-precision, three-part correction routine established by Bellini et al. (2011).

Besides the measured pixel positions of all detected objects, instrumental magnitudes, and the geometricdistortion corrected coordinates, the codes mentioned in

a much better dynamic range than that of the WFPC2 due to the better resolution of analog-to-digital converter: 16 bits versus 12 bits for WFPC2. 
this section deliver an important quality parameter of the ePSF fit to each image - qfit - the sum of absolute differences between the observed pixel values and the model, divided by the total flux. It characterizes the degree of mis-match between the fitted ePSF and the actual profile over the central portion of a source. The qfit can be as low as 0.01. Fainter sources with qfit $>0.5$ appear to be too noisy for accurate astrometry. In addition, we used a plot of qfit vs. instrumental magnitude (see Fig. 4) to cull apparent artifacts such as cosmic rays by defining an empirical curve which separates genuine stars from numerous false detections. Extended sources such as external galaxies having intrinsically higher qfit values might be lost in this partition, but those are not targets of our study. A few surviving contaminants were identified in the later cross-idenfications among the overlapping frames.

\subsection{Astrometric Reference Frame}

The standard practice in working with partially overlapping small images of the sky is to build an overarching astrometric reference frame, covering the entire FOV. This was achieved by hierarchical accumulation of distortion-corrected positions from all long-exposure ACS/WFC and WFC3/UVIS frames of GO-12499, starting with subsets of the largest-overlap frames to make initial tiles and expanding them up to a final link between the positions from both $H S T$ imaging instruments. In all solutions, we used only the well-measured stars with instrumental magnitudes in the range $-14<m_{\mathrm{F} 775 \mathrm{~W}}<$ -10 .

First, for each set shown in Fig. 2, we created 15 local catalogs (tiles) into the system of each sub-pointing $B$ (Fig. (3) by applying a linear three-term polynomial leastsquares solution in each coordinate and then averaging the common positions. Next, we chose tile 4 and 12 (corresponding to the numbered sets in Fig. 2) for ACS/WFC and tile 3 and 12 for WFC3/UVIS as starting tiles for building a total of four strips, extending from South-West to North-East. Then, for ACS/WFC, a strip encompassing tile 4 was linked to the system of coordinates of tile 12. Similarly, for WFC3/UVIS, a strip encompassing tile 12 was translated into the system of coordinates of tile 3. Finally, the entire positional catalog of ACS/WFC was translated into the system of WFC3/UVIS catalog. This sequence of building a composite catalog ensures that the WFC3/UVIS tile 3, which covers the central part of star cluster R 136, is a "touchstone" of our global coordinate system. While all listed above leastsquares solutions were linear, the last transformation into the system of WFC3/UVIS catalog required two additional second-order terms. Without these terms, the offset "center-to-edge" between the positions from the two instruments reached up to $\sim 0.6$ WFC3/UVIS pixel. It is suspected that this offset may have its origin in the ACS/WFC distortion-corrected coordinates. Lastly, the global coordinate system, rooted in tile 3 , was rotated around the point corresponding to $\mathrm{RA}=5^{\mathrm{h}} 38^{\mathrm{m}} 15^{\mathrm{s}} .0$ and $D e c=-69^{\circ} 08^{\prime} 37^{\prime \prime}$ so that the rotated pixel axes are aligned with the two axes of equatorial coordinates to within 1'.5. Our final astrometric reference catalog contains 64,396 objects, each with 1-12 detections depending on the number of frame overlaps, with the average of four detections per star.
Table 2 shows the accuracy of various solutions in the sense of an rms scatter in the residuals. The first column lists the name of the HST imaging instrument. The second column indicates the type of area involved in a solution. We note that a "tile-strip" area for WFC3/UVIS is represented by the two adjacent averaged tiles. A strip includes at least 7 tiles and is more sensitive to largescale positional errors. Small dithers within a tile make a tile solution sensitive to the remaining small-scale geometric distortion. The mean rms for the $X$ and $Y$ coordinates are listed in columns 3-4. The number in parentheses gives the rms scatter of these mean values in hundredths of a milli-arc-second. The total number of least-squares solutions, $\mathrm{N}_{\mathrm{sol}}$, is given in column 5 and the average number of common stars per solution is provided in last column. The last line shows the astrometric performance of both instruments relative to each other. For both instruments, Table 2 indicates that the highest accuracy at $\sim 0.6$ and $\sim 0.5$ mas is achieved in the "tile-strip" solutions. This corresponds to $\sim 0.01$ pixel. Remarkably, solving the ACS/WFC catalog into that of WFC3/UVIS produces reasonably high accuracy, which is a clear sign that the applied corrections for geometric distortion in each instrument are as precise as $0.5-1$ mas, with the exception of the noted quadratic differences. The residuals of the last solution as a function of global pixel coordinates are shown in Fig. 5. There are small semi-periodic systematics with a typical amplitude of $\sim 0.02$ WFC3/UVIS pixel equal to 0.8 mas on the sky. Currently, this is state-of-the-art $H S T$ differential astrometry over scales of $\sim 15^{\prime}$. Differential astrometry over smaller scales (e.g., in the cores of globular clusters) requires fewer calibrations and transformations, and can therefore achieve even higher accuracies.

\subsubsection{Intricacies of astrometry with WFPC2}

The WFPC2 camera was the first HST imaging instrument on which the ePSF concept was successfully developed for undersampled images (Anderson \& King 2000). Contrary to governing wisdom at the time, it was shown that undersampled images can provide extremely precise stellar positions so long as the constructed PSF accurately represents a star's flux distribution on the detector. It also solved the nagging issue of pixel-phase errors, which inevitably appear in the case of an inadequate model PSF, such as a Gaussian curve (e.g., Anderson \& King 2000). For all WFPC2 _c0f .fits type frames, we used the library ePSF in a $3 \times 3$ configuration over each of the WFPC2 chips Anderson \& King 2000) implemented in the code dubbed img2meta. This software includes accurate correction for geometric distortion for each of the WFPC2 chips (Anderson \& King 2003). The library ePSFs are available for the following filters only: F300W, F336W, F439W, F555W, F606W, F658N, F675W, and F814W. In the case of a missing library ePSF, we used the available nearest-in-centralwavelength ePSF. An open issue for the WFPC2 imager is the potential bias in positions due to the timedependent CTI effect, whose photometric calibration is provided by Dolphin (2000, 2009). While the effects of CTI in photometry with WFPC2 have been monitored for years (e.g., Golimowski \& Biretta 2010), to date no detailed report discussing possible CTI effects on astrometry with WFPC2 is available. Given that the CTI 
mostly affects faint sources and that the VFTS stars of primary interest to our study are intrinsicallly bright, we chose to neglect these effects for now. An additional argument in favor of ignoring the CTI in WFPC2 is the lack of a perceptable slope in our LMC proper motions as a function of magnitude (Sect. 3.3). However, such a slope might be mitigated but not eliminated, if the distribution of HST roll angles for WFPC2 frames is reasonably random.

The software for both ACS/WFC and WFC3/UVIS provides the distortion-corrected pixel coordinates of sources in metachip format; that is the pixel coordinates from each of the two CCD chips are transformed into a single system of coordinates, which then can be readily used in building an astrometric reference frame or in calculating proper motions. The WFPC2 has four separate cameras which are inherently prone to gradual displacement (Anderson \& King 2003). The conclusion of these authors is that the interchip separations cannot be predicted with accuracies better than $\sim 0.2 \mathrm{WF}$ pixel. Also, the inner edges of the WFPC2 CCD chips are strongly vignetted and must be excluded from all datasets (Gonzaga et al. 2010, see Table 1.2).

We further explored the feasibility of constructing a metachip frame by calculating the chip constants (coordinate offsets $c x, c y$, and rotation angle $\Theta$ around the point $x=425 y=425$ pixels) using the prescription of Platais et al. (2002, 2006) and the astrometric reference frame, described in Sect. 3.2. Following this algorithm, first we found the best reference stars from leastsquares solutions of each chip into the reference frame using only a linear three-term polynomial for each of the axes. Then, these pre-selected reference stars were used in a global least-squares solution using only the linear terms for each of the axes but this time for all chips simultaneously. Prior to this global solution the scales of all chips were adjusted to that of chip WF3, which is the adopted reference chip for the WFPC2 camera. The chip constants were found by iterative sampling in the $(c x, c y, \Theta)$ space for each chip (starting from PC1 to WF4, WF2, and ending with WF3) and searching for a global minimum of $\chi^{2}$. Minimization was stopped when the change in $c x$ and $c y$ was less than 0.002 pixel and in $\Theta$ - less than 0. . 5 . To calculate the mean chip constants, we used 219 WFPC2 frames ranging in time from 1994.004 to year 2003.1313. A variable portion of the chip constants $\Delta G a p_{X}, \Delta G a p_{Y}, \Delta R o t$ with respect to chip WF3 is shown in Fig. 6] as a function of time. We conclude that the angular orientation of the chips is stable over the considered period of time. A rather large scatter between selected epochs mainly reflects the uncertainties due to the varying number of reference stars. However, there are apparent changes in relative positions between the chips that is a non-linear function of time. There are also some smaller $\sim 0.2$ pixel fluctuations in the chip separations. The pattern of changes and their numerical values are in excellent agreement with the results of a similar study by Anderson \& King (2003). It is interesting to note that the gap evolution for WF2 and WF4 appears to be identical (in Fig. 6] compare $\Delta G a p_{X}$ for WF2 with $\Delta G a p_{Y}$ for WF4), considering the swap of a readout direction. Unfortunately, our data are seven years short of the WFPC2 decommissioning time in 2009. An unpublished study
(L. Bedin, private communication) indicates that there is a turnover in the gap expansion around the year 2004. Therefore, the incomplete curves shown in Fig. 6] cannot be extrapolated beyond the year 2003. In terms of proper motions with WFPC2, there are two alternatives: 1) pixel positions from each chip could be considered independently; or 2) one could construct an astrometricgrade global (metachip) coordinate system from all four CCD chips using the chip constants, inferred from the same frame. In the case of linear transformations that are using a three-term polynomial, both approaches produce similar levels of astrometric accuracy. Considering this, we opted for the simpler and more straightforward independent-chip approach.

The multiple archival images of the 30 Dor region obtained with WFPC2 provide an opportunity to examine the high-frequency part of the geometric distortion for each of the four chips, again with respect to the astrometric reference frame. This is facilitated by the very small proper motions of individual stars in the LMC and the anticipated tiny positional displacements over a nearly two-decade extent of the archival WFPC2 epochs. This time we accumulated residuals from linear solutions for stars brighter than instrumental magnitude -5.5 and not exceeding the averaged residual per each WFPC2 pixel by more than 0.1 for PC1 and 0.2 pixel for all WF cameras, accordingly. Since the astrometric reference frame is on the system of WFC3/UVIS pixels, residuals (and the following 2-D maps) are necessarily in the same system. Similar to Anderson \& King (2003), we averaged residuals on a $17 \times 17$ grid with steps of 47 pixels and in bins with a radius of 42 pixels from each gridpoint. If a bin contained less than 50 residuals, it was discarded, thus resulting in empty bins near the corners of chips and in the vignetted parts of chips (Fig. 7). The averaged residuals indicate locally-correlated patterns which is a hallmark of the residual high-frequency component in the geometric distortion. On the system of chip's native pixels (as measured and corrected for geometric distortion), even the largest averaged residuals do not exceed $\sim 0.03$ pix. Therefore, the derived spatial maps of residual geometric distortion are useful mainly for studies targeting extremely high astrometric precision, such as are presented here. We subtracted the inferred residual geometric distortion from the WFPC2 pixel coordinates prior to calculating proper motions. We note that at this level of precision other effects, e.g, the filter-dependent part of geometric distortion, might also become significant, but we have not explored this.

Finally, the same residuals from the linear solutions of WFPC2 frames into the astrometric reference frame were used to obtain the curves of expected standard positional error as a function of instrumental magnitude. Due to the high similarity of centering errors among all three Wide Field cameras of WFPC2, their residuals were combined. The residuals were binned by magnitude and in each bin a median was calculated, which then was converted into the estimate of standard deviation by applying a factor of 1.4825. The resulting curves of expected standard error are given in Fig. 8. Although these residuals include proper motions, their contribution appears to be insignificant due to the remoteness of LMC and the very low expected apparent velocity dispersion in the tangential plane. 


\subsection{Proper Motions}

With the benefit of having a comprehensive astrometric reference catalog, generated from our long-exposure frames of GO-12499, it is now possible to link any existing frame in the region of 30 Dor to this catalog. For the sake of convenience in finding the common stars, we supplemented the catalog with missing bright stars $\left(m_{\mathrm{F} 775 \mathrm{~W}}<-14\right)$ from Sabbi et al. (2013). Our reference catalog is in the system of distortion-corrected pixel coordinates of frame ibsf03irq taken with WFC3/UVIS. First, we used the global distortion-corrected pixel positions and rotated them to match closely the RA and Dec directions. Second, the J2000 equatorial coordinates were calculated for a total of 67,391 stars down to instrumental magnitude of $m_{\mathrm{F} 775 \mathrm{~W}}=-10$ that define our reference catalog. The corresponding solutions into the UCAC3 positions (Zacharias et al. 2010), using a linear 3-term polynomial and a total of 623 common reference stars in each coordinate, produced standard deviations $\sigma_{\mathrm{RA} \cos (\mathrm{Dec})}=0$ ". 120 and $\sigma_{\text {Dec }}=0$ ". 117 . We note that in the region of the LMC, the latest UCAC4 catalog (Zacharias et al. 2013) has no improvement over the UCAC3 because the ground-based proper motions of the LMC stars are too noisy over small areas such as the 30 Dor. Then, the fits-header information of each target frame was used to calculate equatorial coordinates at the vertices of the metachip's FOV. These coordinates allowed us to select a well-defined list of link stars, enabling the transformation of a target frame into the system of a reference catalog.

\subsubsection{Finding Common Stars}

In order to find the common stars between a chosen frame and a list of link stars, we used the so-called vectorial differences in the pixel space drawn from the brightest 100-150 stars in both lists: the matching objects produce parallel vectors that appear as a significant clump in the $2-\mathrm{D}$ vector space among the randomly distributed false matches. A total of $150^{2}=22500$ vectors can be generated for a sample of the 150 brightest stars, which appears to be a good compromise between the required computational time and a reliability of the clump's detection. The 2-D search for the vectorial clump was substantially sped up by working with sorted arrays of vectors. Once a clump of matching stars is identified, the next step is to produce an initial least-squares solution (normally, using a 3-term linear polynomial model in each coordinate) and then to improve it by including all stars with reliable positions. The last set of polynomial coefficients (including all significant higher-order polynomial terms) is then used for the final transformation of a target frame into the system of a reference frame, which is a basic step in deriving proper motions.

The technique of vectorial differences is extremely robust and works for heavily contaminated samples and/or small fractions of overlapping areas. There are, however, two preconditions for a success: i) the scale must be the same in both systems of pixel coordinates, and ii) the axes should be collinear. The first condition is trivial to satisfy by applying an appropriate scale factor. The second can be less tractable, especially for cases of an arbitrary roll angle of $H S T$ in combination with a low fraction of common stars. For sheer simplicity and con- clusiveness, we chose to sample all possible angles between the two coordinate systems, from $0^{\circ}$ to $360^{\circ}$ at 0.5 steps (Fig. 9). This also provides a means to statistically estimate the significance of a detected clump. The software developed for this analysis has two modes: a) an interactive mode, enabling a visual check of the residual plots and allowing to toggle polynomial terms, which is helpful in handling complicated cases; b) an unattended automatic mode, to process large numbers of frames. The output consists of $X Y$ pixel coordinates in the system of the astrometric reference catalog and the measured apparent magnitude for all objects qualified as real detections (see Fig. 4).

For this study, there are a total of 149 ACS/WFC and WFC3/UVIS first-epoch frames (images), thus yielding one catalog for each frame. All combined, these constitute a global master list of 396,616 objects in the area of 30 Dor with $14.5<m_{\mathrm{F} 775 \mathrm{~W}}<25.5$. Apparent magnitudes in VEGAMAG system are obtained by adding a photometric zeropoint listed in HST Data Handbooks for each of the instruments. No correction is applied for the differences in the aperture size. A star in the master list may have a single or up to 17 multiple detections (found within the radius of two WFC3/UVIS pixels), which is determined by the frequency of frame overlap at the location of this star. Multiple detections allow us to calculate the mean position, its standard error in each coordinate, and to exclude apparent outliers. The latter were identified by comparing the estimated error at a given instrumental magnitude with the actual offset from the mean position. We emphasize that our master catalog has a high degree of completeness, with the exception of brightest stars which are overexposed on all available frames, and some fainter stars located too close to a bright star. Such a master catalog is handy to quickly identify any star on any image but it is not a substitute for an astrometric reference frame.

A total of 174 selected archival WFPC2 frames were similarly translated into the system of the astrometric reference catalog, to produce 174 catalogs. As indicated in Sect. 3.2.1. construction of the latter was done for each chip independently, using a linear transformation into the reference catalog. The number of available reference stars per chip ranges between 6 and $\sim 1000$, nearly independent of chip selection and despite the fact that the PC1 covers a factor of four smaller area of the sky than the remaining WFPC2 chips. There is only a handful of shortest 3-5 s exposures through the broadband WFPC2 filters, thus limiting the number of available reference stars per chip to less than 15 stars. We note that $22 \%$ of the initially selected WFPC2 frames in Sect. 2.1 yield unsatisfactory astrometric accuracy and were not used in calculation of proper motions. In general, our linear transformations appear to be well-constrained, which is demonstrated by the fact that the average standard error of a solution is small - at the level of 0.09 WFC3/UVIS pixel (equivalent to $0.04 \mathrm{WFPC} 2 / \mathrm{WF}$ pixel).

\subsubsection{Calculating Proper Motions}

Once all positions in all exposures are on a system of the astrometric reference catalog, selecting epoch observations for a star and the subsequent calculation of its proper motion is straighforward. At each position from the global master list, we checked which of the 
$\sim 3.2$ million detections are within two WFC3/UVIS pixels around this position?. For all identified detections around each selected position from the global master list, first, a linear unweighted least-squares fit was applied to the $X$ and $Y$ positions as a function of time, provided the epoch span was larger than 3 years and at least 4 detections were found. The slope from this fit is the proper motion and the standard deviation of the slope is the error of proper motion. Although the $X$-axis in the astrometric reference catalog is aligned with Right Ascension, it has the opposite direction, thus requiring a sign flip for the calculated proper motion in $X$. Each linear fit was tested for potential outliers and the most deviant position was eliminated if its departure from the fit exceeded $4 \sigma$. The solution was then repeated and the residuals again checked for a possible outlier, if the limitation of 4 datapoints had not been reached and/or the total number of deviant points is not higher than three. In fact, even three rejected datapoints signal that there might be an image confusion issue and such a star may require a closer examination.

There was some reasoning behind our decision to initially use unweighted positions in the fit. First, our main targets are intrinsically bright $\mathrm{OB}$ spectral-type stars which are never under-exposed even on very short 3-5 s exposures through the F555W and F814W filters of WFPC2. Effectively, these stars have the accuracy of measured positions dominated by systematics with a negligible contribution by Poisson noise. This is different for faint stars for which shot noise dominates and can be estimated. Second, the epoch distribution of observations is highly uneven and often nearly all positions at a middle epoch are slightly displaced by $\gtrsim 0.05$ WFC/UVIS pixel with respect to an overall trend in the positions with time. In addition, the spread of datapoints at the early epochs sometimes reaches a total range of $0.2-0.3$ WFC3/UVIS pixels. These findings hint to possible systematics in the WFPC2 frames. Such cases can be identified by the larger-than-normal proper-motion errors for a given number of available observations.

Despite the robustness of unweighted least-squares fits, there are a couple of noteworthy caveats: i) no differentiation is given to the datapoints having clearly disparate positional accuracies, owing to significant differences in signal-to-noise ratio; ii) the presence of potential systematics is only loosely indicated by somewhat elevated proper motion errors at a given stellar magnitude. These issues can be mitigated by "equalizing" the datapoints, that is, using their weights. For this, we used the curves of expected standard errors described in Sect. 3.2.1 and given in Fig. 8. The presence of $a$ priori error estimates also allows us to calculate $\chi^{2}$ and the goodness-of-fit probability $Q$ (subroutine GAMMQ in Press et al. 1992). In our calculations of proper motions, a low probability $Q$ can be due to underestimated random errors of positions and/or the presence of systematic errors in the positions. The latter point is illustrated by Fig. 10 which provides the weighted fit to the positions of a well-measured brighter star. This star has a total of five

\footnotetext{
7 A two-pixel-large displacement over 20 years would correspond to $\sim 1000 \mathrm{~km} \mathrm{~s}^{-1}$ at the distance of the LMC. This restriction may end up excluding Galactic stars with relatively high proper motions projected onto the 30 Dor region.
}

tightly-clumped positional measurements on ACS/WFC frames in 2011.7. However, the dispersion of WFPC2 measurements, obtained with all three WF cameras, is higher by nearly an order of magnitude, consistent with the notion that at brighter instrumental magnitudes the WFPC2/PC1 produces three times less accurate positions than the ACS/WFC and WFC3/UVIS, but the WF cameras - even $\sim 6$ times worse. In addition, the observations with WF2 (epoch=1999.7) in $Y$-coordinate show a systematic offset comparing to the observations obtained with WF4 (epoch=2000.7). Despite these systematics, the calculated proper motion, $\mu_{X}=+0.20 \pm 0.02$

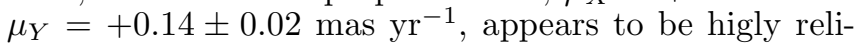
able, also indicated by the the goodness-of-fit probability exceeding 0.95 .

Conversely, stars with significantly fewer measurements are more susceptible to such systematics and often have very low probability $Q$. We noticed that in the areas of heavy overlap of ACS/WFC and/or WFC3/UVIS frames - as a rule at the edges and corners of chip's FOV (see Fig. 2) - the epoch 2011.7 observations tend to produce significantly higher dispersion (e.g., Fig. 5) than the predicted standard errors would indicate. This inflates the $\chi^{2}$ and, via the probability $Q$, marks the propermotion calculation as unreliable. In addition, the propermotion errors in such cases can be underestimated. One way to mitigate these effects would be to rescale the expected standard errors of ACS/WFC and WFC3/UVIS at brighter magnitudes and optimize the $\chi^{2}$, especially for stars with numerous measurements (e.g., $n>25$ ). An alternative could be to provide both solutions, with and without weights. Normally, with sufficient number of observations, such solutions differ insignificantly, modulo the error estimates. Closing discussion on possible systematics, we note that in our WFPC2 frames the socalled pixel phase error, due to the variations of ePSF over time, is contributing to the error budget at levels less than $\sim 0.02 \mathrm{WFPC} 2 / \mathrm{WF}$ pixel, which is in agreement with conclusions by Anderson \& King (2000) about this effect.

The final catalog of positions and proper motions is based on the weighted fits. The upper limit of acceptable proper-motion precision in our catalog of 86,590 stars is adopted at $\sigma_{\mu} \sim 1.5$ mas $\mathrm{yr}^{-1}$. As expected, proper motions derived from fewer detections $(n<9)$ appear to have higher errors. However, nearly a half of the highprecision proper motions $\left(\sigma_{\mu}<0.3 \mathrm{mas} \mathrm{yr}^{-1}\right)$ are based on a smaller number of datapoints (Fig. [11). Such cases should be considered to be less reliable. We expect to reanalyze all problematic and low-accuracy-yielding cases later with the aid of the GO-13359 second-epoch observations.

\subsubsection{Exploration of local corrections}

As shown in Fig. 5 residuals from a transformation of the global ACS/WFC pixel coordinates into the WFC3/UVIS global coordinates show small coordinatedependent systematics. Even if the positions from WFPC2 observations were perfect, such systematics would show up in the proper motions to the extent that our linear transformations cannot account for them. One common practice to deal with such systematics is to apply a local adjustment to each star (e.g., Anderson \& van der Marel 2010). It is expected that 
there is a characteristic scale-length over which the variations due to spatial systematics are negligible. For ACS/WCS, a 100 pixel box (equal to $5^{\prime \prime}$ ) was adopted by Anderson \& van der Marel (2010) as such a scale-length. In order to eliminate systematics from proper motions, the local stars must be drawn from a population with physically consistent bulk motion (e.g., a star cluster), so that the differences in proper motions among all used local stars are smaller than the sought-after systematics. We explored how well this might work for the 30 Dor region, where the spatial density of stars varies by orders of magnitude, from the center of R 136 out to the LMC field stars interspersed with filaments of dust and molecular gas. First, we chose only the 11 nearest stars with $m_{\mathrm{F} 775 \mathrm{~W}}<23 \mathrm{mag}$ and proper motion error less than

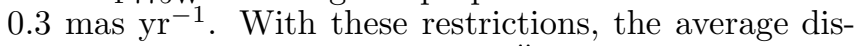
tance of the outermost star is $\sim 4^{\prime \prime}$, albeit in some cases it can be as large as $12^{\prime \prime}$. Then, the median proper motion of these local stars, that is the local correction, was subtracted from that of a target star. To evaluate the impact of local corrections, we selected a small sample $(n=50)$ of VFTS stars with high proper motion acuracy $\left(\sigma_{\mu}<0.1\right.$ mas $\left.\mathrm{yr}^{-1}\right)$ and estimated the dispersion of their proper motions before and after the correction. We did not find an appreciable decrease in the dispersion of locally-corrected proper motions and, therefore, opted not to apply these corrections to our proper motions.

\subsection{Poleski et al. Proper Motions}

Recently, Poleski et al. (2012) published a propermotion catalog of over 6.2 million stars in the direction of both Magellanic Clouds. The authors used groundbased observations spanning 8 years and obtained $\sim 400$ 700 individual epochs per star. The formal errors of the derived proper motions can be as low as 0.2 mas $\mathrm{yr}^{-1}$, thus making them potentially attractive from the standpoint of potential runaway stars. Nearly $75 \%$ of VFTS stars are present in this catalog, all with $I$-magnitudes ranging from 13.0 to $17.0 \mathrm{mag}$. In this magnitude range, the Poleski et al proper motions have their estimated uncertainties better than 0.3 mas $\mathrm{yr}^{-1}$. We found 50 common VFTS stars between Poleski et al. and our proper motions with mutually comparable formal uncertainties. If the Poleski et al. error estimates are correct, we would also expect similar proper-motion dispersions, which are dominated by the measurement errors. The distribution of measured line-of-sight velocities for the dominant stellar populations in the LMC indicates an upper limit of the intrinsic velocity dispersion at $\sim 30 \mathrm{~km} \mathrm{~s}^{-1}$ (van der Marel et al. 2002), corresponding to $\sim 0.1$ mas $\mathrm{yr}^{-1}$ at the distance of the LMC. We note that the systemic rotation of the LMC has no contribution to the intrinsic velocity dispersion over the small size of the 30 Dor field. A star-by-star comparison (Fig. 12)

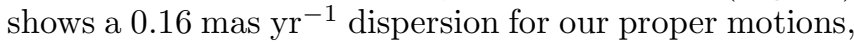
consistent with the estimated proper-motion errors. In contrast, the same stars in the Poleski et al catalog show a large dispersion at the 0.8-1.3 mas $\mathrm{yr}^{-1}$ level, depending on whether or not the obvious outliers are eliminated. This indicates that their proper motion errors for relatively bright stars are underestimated by a factor of three or more. With such high actual measurement errors, the proper motions from Poleski et al. (2012) appear to be inadequate for studies of internal velocities in the Magellanic Clouds.

\section{DISCUSSION AND APPLICATIONS}

Our main goal is to detect OB runaway stars and identify a likely site of their origin using our new proper motions. A more general approach, however, would be to identify runaway stars without applying any prior knowledge of photometric, spectral and kinematic characteristics of stars and then focus on the most interesting cases. This approach is not straightforward with the current data due to the incomplete coverage of the FOV and a significant variation in the accuracy of proper motions across the field caused by the uneven distribution of the first-epoch WFPC2 frames. Therefore, we adopted a mixed approach: the statistical properties of our proper motions are based on a selection of VFTS stars, which are spectroscopically confirmed OB stars, but possible runaways are searched among all brighter stars with proper motions.

\subsection{Characterizing the global proper motion accuracy}

As indicated in Sect. 2.1 and Table 1 the distribution of WFPC2 archival frames is patchy and the combined spatial coverage is only $\sim 30 \%$ of the size of our astrometric field (Fig. 13). Our proper motions show no perceptible presence of spatial- or magnitude-related systematic errors (Fig. 14 top panel). The proper-motion distribution narrows significantly if only the higher-precision proper motions are plotted (Fig. 14 bottom panel). In the accuracy range $\sigma_{\mu}<0.35{\text { mas } \mathrm{yr}^{-1} \text { and at } m_{\mathrm{F} 775 \mathrm{~W}}<}$ 18, there are a total of 1262 stars. Among them, a handful of stars have proper motions exceeding 1 mas $\mathrm{yr}^{-1}$ in either coordinate, which we excluded from the analysis. At the LMC distance modulus $m-M \approx 18.50$, a proper

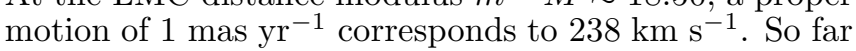
the studies of LOS velocities for the OB stars indicate an upper runaway velocity at $\sim 100 \mathrm{~km} \mathrm{~s}^{-1}$ (Evans et al. 2015). Hence, it appears unlikely that proper motions

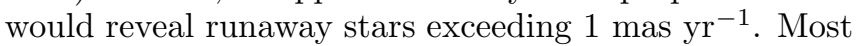
likely these fast-moving stars are just the foreground Galactic stars. Also, with only two distinct epochs available for these stars, we are not able to confirm their large proper motion yet.

In our following examination of derived proper motions we focus entirely on brighter stars $\left(m_{\mathrm{F} 775 \mathrm{~W}}<18\right)$. This sample is scrutinized in two complementary ways: i) we estimate the bulk properties of these stars; ii) we check the proper-motion fit of selected stars. Finally, we searched for possible OB runaway stars and estimated the degree of success in finding them. In this magnitude range, there is a total of 285 VFTS stars with measured proper motions. Most of them have formal errors less than 0.2 mas $\mathrm{yr}^{-1}$, however, $38 \%$ of these VFTS stars have less than 9 datapoints per star. As indicated in Sect. 3.3.2, a small number of datapoints may produce relatively poor proper motions. Therefore, we selected a sample of 109 VFTS stars, requiring that the number of datapoints be greater than eight, the propermotion error in both axes is $\sigma_{\mu} \leq 0.1$, and the goodnessof-fit probability $\gtrsim 0.1$. The vector-point diagram of this sample is shown in the top panel of Fig. 15. Then, these proper motions, separately in each coordinate, are 
convolved with a unity Gaussian of the width equal to the proper-motion error (never allowing it to be smaller than 0.02 mas $\left.\mathrm{yr}^{-1}\right)$. These Gaussians are summed up and the sum is roughly normalized (bottom panels of Fig. 15). The derived smooth distributions are then fit with a Gaussian. This provides an important characteristic of our best proper motions. In both axes, the rms scatter around zero proper motion is $\sigma_{\mathrm{pm}}=0.10 \mathrm{mas} \mathrm{yr}^{-1}$. This can be converted into the observed velocity dispersion. Thus, the observed dispersion of transverse velocities is $\sim 25 \mathrm{~km} \mathrm{~s}^{-1}$. This is a factor of $\sim 3$ larger than the line-of-sight velocity dispersion derived from single VFTS stars (Sana et al., in preparation), but similar to the internal velocity dispersion of the old LMC disk stars van der Marel et al. 2002; van der Marel \& Kallivavalil 2014). While we are not able to resolve the internal velocity dispersion of either 30 Dor or the LMC disk, our proper-motion measurement accuracies appear to be sufficient to identify possible runaway stars.

Finally, a good external check of our proper-motion accuracy is provided by the mean measured LOS velocities of VFTS stars. In order to convert them to the equivalent proper motions, first, the global mean LOS velocity of $268.5 \mathrm{~km} \mathrm{~s}^{-1}$ was subtracted from each individual velocity. We note that the bulk of $\mathrm{OB}$ stars in the 30 Dor region are mainly concentrated in and around R 136 (NGC 2070) and the OB association NGC 2060 (see Fig. 1). There is a $\sim 10 \mathrm{~km} \mathrm{~s}^{-1}$ difference in the mean LOS between these two major stellar nurseries. Therefore, our adopted mean velocity is just an arithmetic mean and is not representing a particular stellar system in 30 Dor. Second, we selected 199 VFTS stars with actual proper-motion errors $\sigma_{\mu} \leq 0.14$ mas $\mathrm{yr}^{-1}$. Then, a conversion factor from linear to angular velocities was applied to the offset LOS velocities from the global mean, yielding equivalent proper motions along the line-of-sight. For these proper motions, we constructed a summed-up Gaussian as in Fig. 15 but this time using the corresponding standard error of each star's astrometric proper motion. The resultant Gaussian is shown in Fig. 16] with its width equal to 0.08 mas $\mathrm{yr}^{-1}$. It is smaller only by $20 \%$ than the similar widths obtained from our best astrometry shown in Fig. 15. The actual rms dispersion of distribution for the 199 VFTS

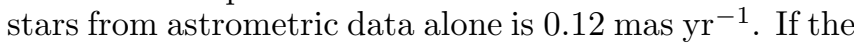
three-dimensional velocity distribution of the stars were roughly isotropic, then one would have expected the two distributions shown in Fig. 16] to be the same. This is not the case, although the distributions are reasonably similar. The fact that the distribution derived from the actual proper motions is somewhat broader indicates most likely that there are small systematic errors in the proper motion data, in addition to random errors. To lowest order, the dispersion of the systematic errors can be estimated to be $\lesssim \sqrt{\left(0.12^{2}\right)-\left(0.08^{2}\right)}=0.09$ mas $\mathrm{yr}^{-1}$. Hence, there may be systematic proper motion errors in this high-quality sample that are comparable to the random errors. This needs to be taken into account when assessing runaway star candidates.

\subsection{Identifying astrometric high-velocity stars}

If we assume that all stars are born in star clusters and associations, then any star with a velocity exceed- ing the cluster's escape velocity can be considered as a dynamically-ejected "runaway". However, the shortlived O stars may escape by another mechanism - via the binary supernova - which can produce much faster runaways. Thus, Blaauw (1961) for Galactic O stars suggests an empirical lower limit of the excess peculiar velocity at $40 \mathrm{~km} \mathrm{~s}^{-1}$. At the distance of LMC this corresponds to a proper motion of $\sim 0.2$ mas $\mathrm{yr}^{-1}$.

To identify fast-moving stars among the sample of brighter stars with $m_{\mathrm{F} 775 \mathrm{~W}}<18$, we selected stars with a calculated proper motion in either coordinate that differs from zero at the $\geq 5 \sigma$ significance level. There are 105 such stars that also have the goodness-of-fit probability $>0.2$ and the number of datapoints greater than eight. The vector-point diagram of these stars is shown in Fig. 17. top panel. A total of 93 of these stars have $V I 8$ photometry from Sabbi et al. (in preparation). A $V I \mathrm{CMD}$ in the region of 30 Dor is provided in the same figure. Note the morphology of the red clump at $1<V-I<2$, which is stretched out due to the differential reddening.

We can divide the sample in color at $V-I=0.9$, which approximately separates young blue stars from old red stars ( $70 \%$ vs. $30 \%$, respectively). The observed fast-moving red stars may simply be outliers in the LMC velocity distribution. The LOS velocity dispersion of old red giants is $\sim 25 \mathrm{~km} \mathrm{~s}^{-1}$ (Cole et al. 2005). This implies that $\sim 10 \%$ of such stars are expected to have $|V|>50 \mathrm{~km} \mathrm{~s}^{-1}$ in one of the two coordinate directions. Therefore, the observed red stars with high proper motions are not of interest in the context of the runaway star phenomenon (although they might be of interest for searches of stripped SMC stars behind the LMC; Olsen et al. (2011)). So, we focus instead on the young stars. These have the intrinsic velocity dispersion of $\sim 10 \mathrm{~km} \mathrm{~s}^{-1}$ (van der Marel \& Kallivayalil 2014), which makes it highly unlikely to find such stars moving in the tail of a Gaussian distribution at $|V|>50 \mathrm{~km} \mathrm{~s}^{-1}$.

We proceeded with a careful examination of every blue and potentially fast-moving star, especially its proper motion fit. First, we separated all fast moving stars into two groups, each associated with the instrument used for the last epoch, that is ACS/WFC or WFC3/UVIS. It was suspected that some residual effect of CTI might be present in either set of observations. Because of a nearly fixed $H S T$ orientation during the observations, this would readily show up in the vector-point diagram as a distinct elongated feature of false proper motions. In fact, there is no such feature visible (Fig. 17, top panel), hence, the residual CTI effects can be ruled out. Second, we examined each proper motion fit and correlated it with a table containing detailed information on each datapoint such as the filter, exposure time, instrumental magnitude, the amount of offset in $X$ and $Y$, the corresponding chip of WFPC2, and the location of the star on this chip. Two main culprits for significant systematic errors were identified: a) the effects of vignetting on WFPC2 chips affect astrometry at least up to 100 pixels from the inner chip edges. Likewise, the outer edges with pixel coordinates ¿750 might be affected by the geometric modelling effects (where they reach the maximum) and, possibly, by

8 The $I$-magnitude, F775W, in our catalog is $\sim 0.2$ mag fainter than that calibrated for infinite aperture in Sabbi et al. 
less accurate corrections of geometric distortion near the edge. We, therefore, opted to eliminate all datapoints from these ambiguous areas; b) systematic offsets also appear to be correlated with a specific chip. We sometimes noticed an unusual scatter if the datapoints are drawn from two or more different chips, while their native pixel coordinates are tighly-clumped on each of these chips. Other factors, such as exposure time may have less contribution to the systematics, but a use of different filters usually increases the scatter of datapoints. On one occasion, we found three stars with a correlated and enlarged proper motion in $Y$, all aligned $\sim 200$ pixels away from one side of a chip. In addition, if prior to a fit, the observations at middle epochs indicate no offset relative to the last epoch observations but the earliest WFPC2 epochs show an obvious offset (hinting at a significant proper motion), such a case was marked as unreliable. Finally, each candidate star was visually inspected on a combined image, constructed from long exposures with ACS/WFC and WFC3/UVIS. Only stars with clean and well-isolated images were kept for the further considerations.

After discarding all stars having the noted peculiarities, we were left with a list of only six possible OB stars with high proper motions. For most of the highproper-motion stars in Fig. 17] we have reason to suspect that the ostensibly high proper motion is due to some residual systematics. This differs from the case for typical stars, for which we found that systematic proper motion errors do not generally exceed the random errors (see Fig. 16]). Our remaining six candidate runaway stars are listed in Table 3 . Notes to this table: magnitudes $m_{\mathrm{F} 555 \mathrm{~W}}(V)$ and $m_{\mathrm{F} 775 \mathrm{~W}}(I)$ from Sabbi et al. (in preparation); Right Ascension (RA) and Declination (Dec) in J2000 coordinates; proper motion in each coordinate and its standard error in mas $\mathrm{yr}^{-1} ; \chi^{2}$ normalized to the number of degrees of freedom, that is, $\mathrm{N}_{\text {frame }}-2$; the goodness-of-fit probability $Q$ ranging between 0 to 1 (low $Q$ indicates a non-Gaussian distribution of residuals and/or a presence of outliers); and the number of frames (epochs) used in the calculation of proper motion. The associated weighted least-squares fits of these six stars are shown in Fig. 18.

Of the six candidate runaway stars in Table 3, three are part of the VFTS survey and the three are not. On average, The VFTS stars appear to have somewhat larger proper motions, up to 0.4 mas $\mathrm{yr}^{-1}$ in one coordinate, while the non-VFTS stars have smaller $\sim 0.2$ mas $\mathrm{yr}^{-1}$ proper motions. In addition, the VFTS stars have only 11-13 datapoints available, while the nonVFTS stars have 30-60 datapoints. This kind of pattern might be indicative of some lingering systematics for these VFTS stars; possibly, their true proper motions could be smaller. In Fig. 15, we marked the candidate LOS runaway OB stars among the selected VFTS stars. Remarkably, only star VFTS 285 is common between the two lists of candidate OB runaways. In light of the noted astrometric systematics, we defer a deeper analysis on the runaway star status, statistics, and confidence levels to the future study encompassing the most recent HST observations.

Since all six stars are located near the rich star cluster R 136, we can now explore whether any of them may have its origin in this cluster. Figure 19 shows the location of all six OB stars from Table 3. The center of R 136 coincides with the zeropoint of the provided gnomonic projection. For convenience, two dotted circles are drawn around this center, indicating the estimates of the halflight and the tidal radius. The former is based on a single-component King model fit to the surface brightness profile (McLaughlin \& van der Marel 2005), while the latter is calculated using a two-component fit in which only the outer component is a King model, thus providing a better fit at large radius (Mackey \& Gilmore 2003). All stars but ID $6=$ VFTS $285(d=2$ '.36) are located within $\sim 1^{\prime}$ from $\mathrm{R} 136$. One of the most intriguing features of the stars in Fig 19 is the orientation of their proper motion vector. Only the likely OB star ID 3 and VFTS 285 show proper motions loosely consistent with the notion that they might be ejected from R 136 with VFTS 285 even being outside the estimated tidal radius of 2 '17. Star VFTS 285 also has an offset LOS velocity $\left(-39 \pm 4 \mathrm{~km} \mathrm{~s}^{-1}\right.$ relative to the systemic velocity of R 136), thus supporting its runaway status. Assuming that R 136 has zero bulk proper motion (not true but estimated to be less than 0.1 mas $\mathrm{yr}^{-1}$ ), our measured proper motion of VFTS 285 translates into $\sim 110 \pm 19$ $\mathrm{km} \mathrm{s}^{-1}$ runaway velocity in the tangential plane at a polar angle of $\phi=-39^{\circ}$ relative to the direction outwards the center of $\mathrm{R} 136$. In terms of runaway velocity, this is consistent with the largest LOS offset velocity identified among the VFTS B-type stars (Evans et al. 2015). However, due to possible remaining systematics in our astrometry, the true proper motion of VFTS 285 might be lower. Therefore, we only tentatively conclude that VFTS 285 appears to be the first likely O-type (classified as O7.5 Vnnn) runaway star in the LMC, detected from proper motions.

In summary, we now have only two reasonable candidate OB proper-motion runaway stars, but a more complete picture should emerge after the planned reductions of the last-epoch GO-13359 observations with HST.

\section{CONCLUSIONS}

We have studied the 30 Dor region in the LMC with the aim of determining individual proper motions of stars. This study is a pilot project for deriving relative proper motions from $H S T$ observations over a significantly larger contiguous area of the sky than ever before. Great attention has been given to constructing the backbone of our astrometric reductions - the astrometric reference frame - by joining separate distortion-corrected global pixel coordinate systems, obtained from our observations with two HST imaging instruments, ACS/WFC and WFC3/UVIS. We were able to limit internal systematics to the level of $\sim 0.02$ WFC3/UVIS pixel (0.8 mas) over scales of $\sim 15^{\prime}$. This was achieved despite the fact that the density of optimal link stars per frame varies by a factor of 6 over the 30 Dor field. We note that a quilt-like pattern of field coverage with various degrees of overlap is a key element in the success of global astrometry over a large FOV.

Next, we explored the potential of using archival WFPC2 frames to derive relative proper motions in the 30 Dor region. A total of 176 WFPC2 frames contributed to this effort. Although they cover only one third of our total FOV, this provides valuable insights on what kind 
of proper-motion accuracies can be achieved with a 17 year time baseline. Our best proper motions near the massive cluster R 136 have formal precision as high as $\sim 20 \mu$ as $\mathrm{yr}^{-1}$. The true proper-motion accuracy is more challenging to quantify, owing to the likely presence of residual systematics. Nevertheless, we show that it is possible to measure proper motions accurately enough to identify fast-moving individual stars in the LMC.

We derived a proper-motion catalog in the region of 30 Dor for 86,590 stars. Its most valuable part is the higher-accuracy portion, listing $\sim 1,300$ stars brighter than $m_{\mathrm{F} 775 \mathrm{~W}}=18$. This list enabled us to select two possible proper-motion runaway stars among a shortlist of six OB stars.

In summary, the main accomplishments of this study are:

1. We provide a detailed description of the construction of an astrometric reference frame based on oneepoch observations with the $H S T$. Internal comparisons indicate a sub-mas accuracy in the positions of our astrometric reference frame over scales exceeding $10^{\prime}$

2. We calculated relative proper motions for large numbers of stars down to $V \sim 25$. This is the first high-accuracy proper motion catalog in the region of 30 Dor, allowing us to search for fastmoving stars using proper motions. Our detailed analysis of a sample of brighter stars showed that the achieved accuracy enables an estimate of individual proper motions, if the true relative proper motion is larger than $\sim 0.1$ mas $\mathrm{yr}^{-1}$.

3. There is a hint that one LOS-velocity O-type runaway star also appears to be a proper-motion runaway. Star VFTS 285 seems to have its proper motion consistent with an origin from the massive star cluster R 136. This will be verified in the future study by adding a final epoch of $H S T$ observations with WFC3/UVIS and ACS/WFC to the existing database.

The authors gratefully acknowledge grant support for program GO-12499, provided by NASA through grants from the Space Telescope Science Institute, which is operated by the Association of Universities for Research in Astronomy, Inc., under NASA contract NAS 5-26555.

Facilities: Hubble Space Telescope

\section{REFERENCES}

Anderson, J., \& King, I. R. 2000, PASP, 112, 1360

Anderson, J., \& King, I. R. 2003, PASP, 115, 113

Anderson, J., \& King, I. R. 2006, Instrument Science Report ACS 2006-01, (Baltimore: STScI)

Anderson, J. 2007, Instrument Science Report ACS 2007-08, (Baltimore: STScI)

Anderson, J., \& Bedin, L. R. 2010, PASP, 122, 1035

Anderson, J., \& van der Marel, R. P. 2010, ApJ, 710, 1032

Bellini, A., Anderson, J., Bedin, L. R. 2011, PASP, 123, 622

Bellini, A., Anderson, J., van der Marel, R. P., et al. 2014, ApJ, $797: 115$

Blaauw, A. 1961, BAN, 15, 265

Chini, R., Hoffmeister, V. H., Nasseri, A., Stahl, O., Zinnecker, H. 2012, MNRAS, 424, 1925

Cole, A. A., Tolstoy, E., Gallagher, J. S., \& Smecker-Hane, T. A 2005, AJ, 129, 1465

Dolphin, A. E. 2000, PASP, 112, 1397

Dolphin, A. E. 2009, PASP, 121, 655

Evans, C. J., Walborn, N. R., Crowther, P. A., et al. 2010, ApJ, 715, L74

Evans, C. J., Taylor, W. D., Hénault-Brunet, V., et al. 2011, A\&A, 530, A108

Evans, C. J., Kennedy, M. B., Dufton, P. L., et al. 2015, A\&A, 574, A13

Hénault-Brunet, V., Gieles, M., Evans, C. J., et al. 2012, A\&A, 545, L1

Golimowski, D. A., \& Biretta, J. 2010, Instrument Science Report WFPC2 2010-04, (Baltimore: STScI)

Gonzaga, S., et al. 2010, in HST WFPC2 Data Handbook, v. 5.0, (Baltimore: STScI)

Gvaramadze, V. V., Gualandris, A. 2011, MNRAS, 410, 304

Hoogerwerf, R., de Bruijne, J. H. J., de Zeeuw, P. T. 2000, ApJ, 544, L133

Kallivayalil, N., van der Marel, R. P., Besla, G., Anderson, J., \& Alcock, C. 2013, ApJ, 764:161

Mackey, A. D., \& Gilmore, G. F. 2003, MNRAS, 338, 85
Mason, B. D., Hartkopf, W. I., Gies, D. R., Henry, T. J., Helsel, J. W. 2009, AJ, 137, 3358

McLaughlin, D. E., \& van der Marel, R. P. 2005, ApJS, 161, 304

Olsen, K. A. G., Zaritsky, D., Blum, R. D., Boyer, M. L., \&

Gordon, K. D. 2011, ApJ, 737:29

Platais, I., Kozhurina-Platais, V., Girard, T. M., et al. 2002, AJ 124,601

Platais, I., Wyse, R. F. G., \& Zacharias, N. 2006, PASP, 118, 107

Poleski, R., Soszyński, I., Udalski, A., et al. 2012, Acta Astron., 62,1

Press, W. H., Teukolsky, S. A., Vetterling, W. T., Flannery, B. P. 1992, Numerical Recipes in Fortran 77 (Cambridge: Cambridge Univ. Press)

Sabbi, E., Anderson, J., Lennon, D. J., et al. 2013, AJ, 146:53

Sana, H., de Mink, S. E., de Koter, A., et al. 2012, Science, 337, 444

Sana, H. 2013, in EAS Publications Series: Setting a New Standard in the Analysis of Binary stars, ed. K. Pavlovski, A. Tkachenko, G. Torres (Cambridge: Cambridge University Press), 147

Stone, R. C. 1991, AJ, 102, 333

Úbeda, L., Kozhurina, V., \& Bedin, L. R. 2013, Instrument Science Report ACS 2013-03, (Baltimore: STScI)

van der Marel, R. P., Alves, D. R., Hardy, E., Suntzeff, N. B. 2002, AJ, 124, 2639

van der Marel, R. P., Anderson, J., Bellini, A., et al. 2014, in ASP Conf. Ser. 480: Structure and Dynamics of Disk Galaxies, ed. M. S. Seigar \& P. Treuthardt (San Francisco, CA: ASP), 43

van der Marel, R. P., Kallivayalil, N. 2014, ApJ, 781:121

Vieira, K., Girard, T. M., van Altena, W., F., et al., 2010, AJ, 140,1934

Walborn, N. R \& Blades, J. C. 1997, ApJS, 112, 457

Zacharias, N., Finch, C., Girard, T., et al. 2010, AJ, 139, 2184

Zacharias, N., Finch, C. T., Girard, T. M., Henden, A., Bartlett, J. L., Monet, D. G., Zacharias, M. I. 2013, AJ, 145:44 


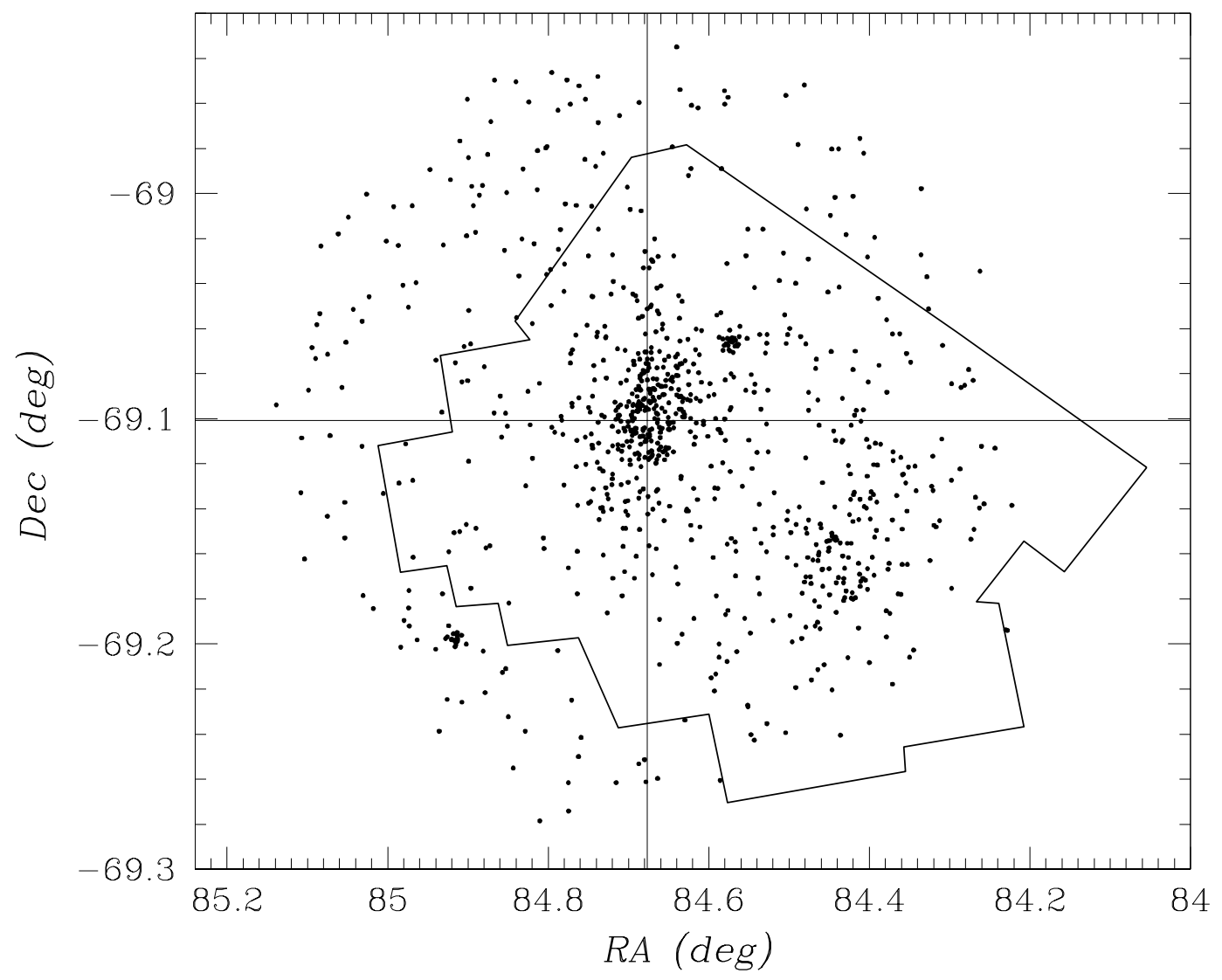

FIG. 1. - Spatial distribution of stars from the VLT-FLAMES Tarantula Survey (dots) and field coverage of the $H S T$ proper-motion survey. The rugged line shows an approximate outer border of the astrometric field. The crosshairs indicate the center of the very massive star cluster R 136. 


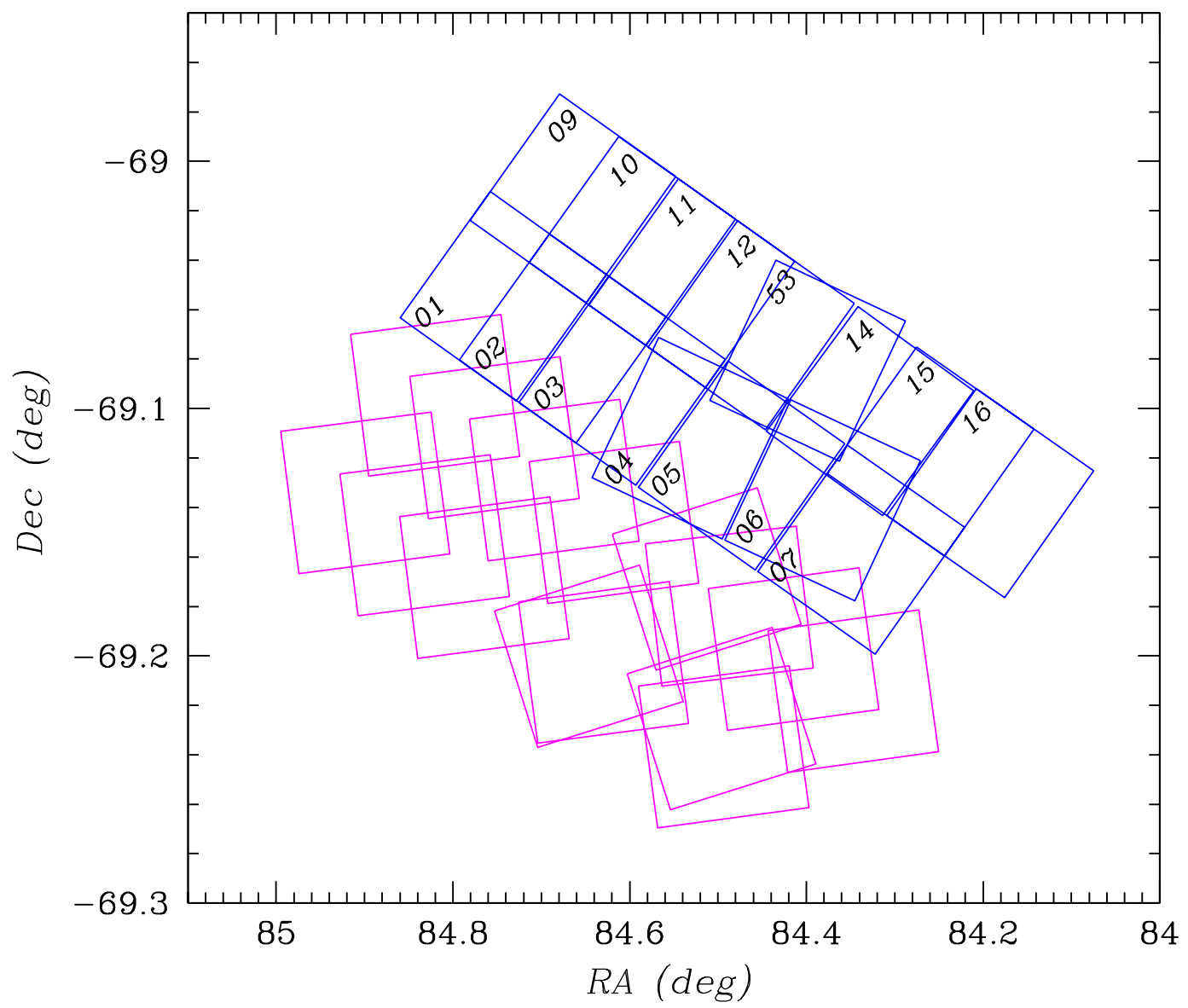

Fig. 2.- Sky coverage obtained with two HST imaging instruments. The Northern semi-rectangular pointing pattern is generated by the primary WFC3/UVIS frames (in blue). Each pointing is marked by the visit number in our HST program. The parallel observations with ACS/WFC are shown in red. The angular size of entire astrometric field is about $13^{\prime} \times 16^{\prime}$. 


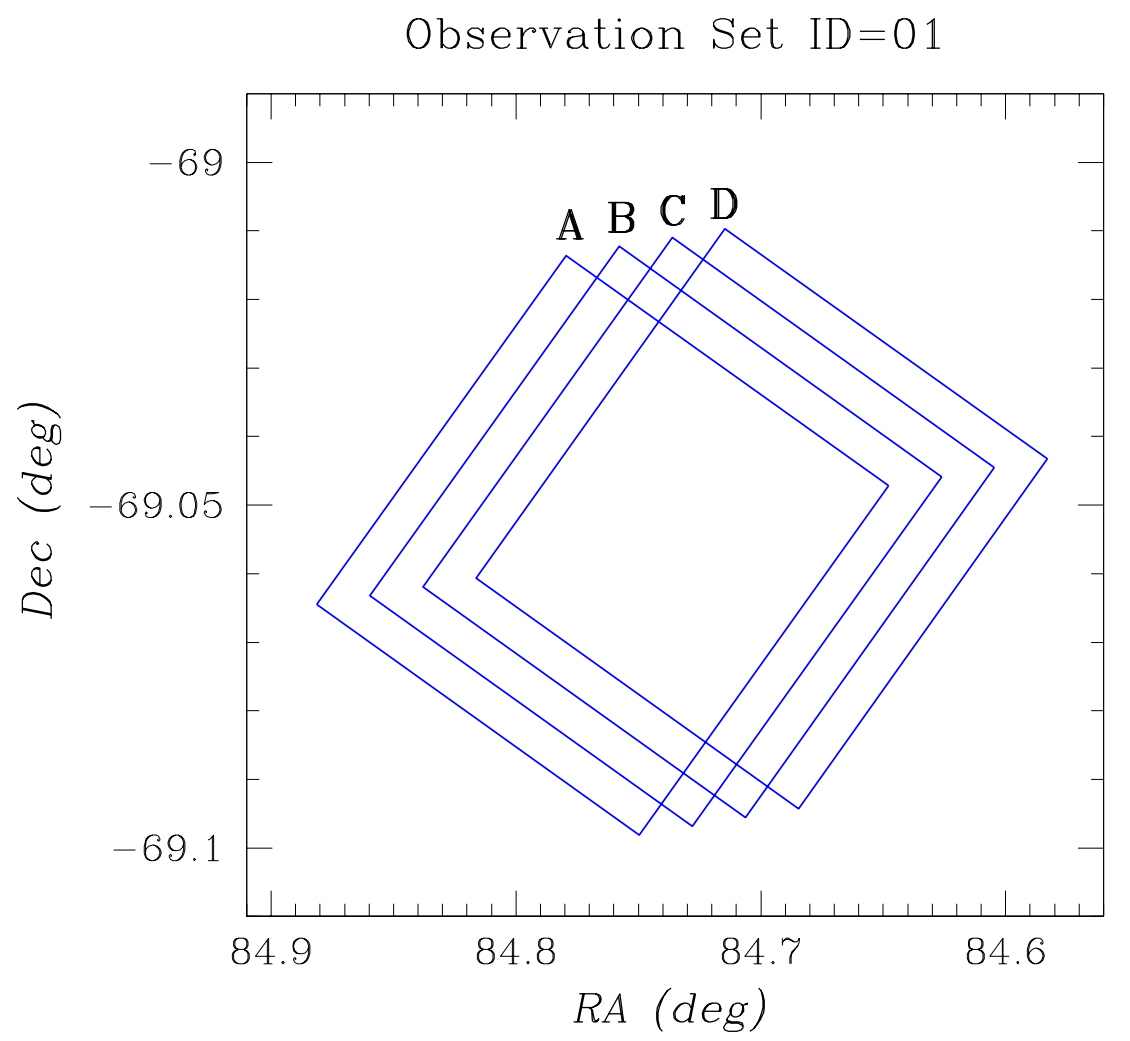

FIG. 3.- Dither pattern used for each observational set. Shown is the observational set 01 taken with the WFC3/UVIS camera during one $H S T$ orbit. The dither step is equal to $10^{\prime \prime}$ along each of the pixel axes. The numbers indicate the sequence of consecutive long exposures. The sub-pointing $B$ was adopted as representative for an entire observational set - all other sub-pointings and exposures were translated into this frame. 


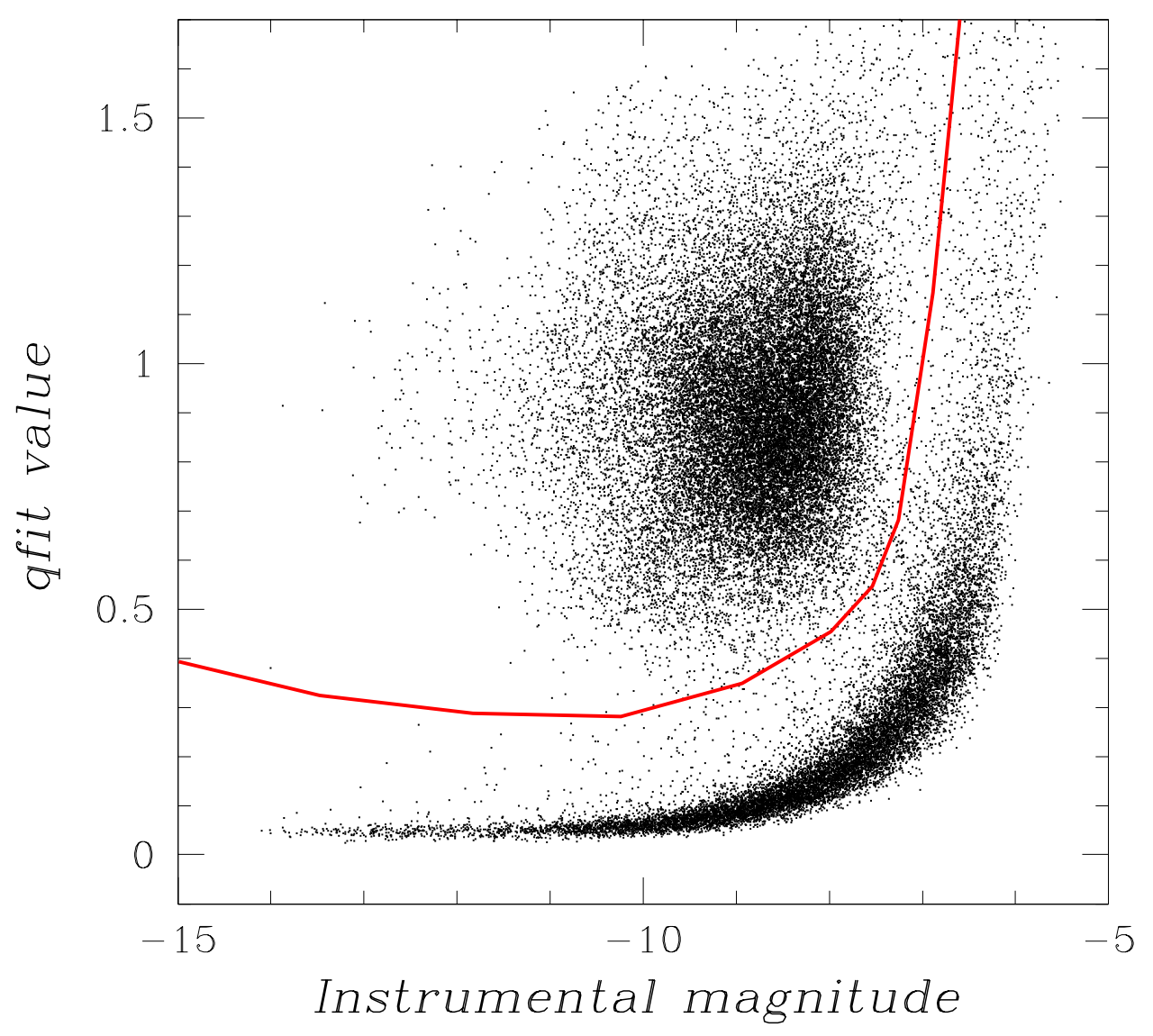

FIG. 4.- Separation of star detections from apparent artifacts in a WFC3/UVIS long-exposure frame. All detections above the red curve were deleted prior to the following astrometric reductions. The same empirical separation curve was used for both $H S T$ imaging instruments: WFC3/UVIS and ACS/WFC. 

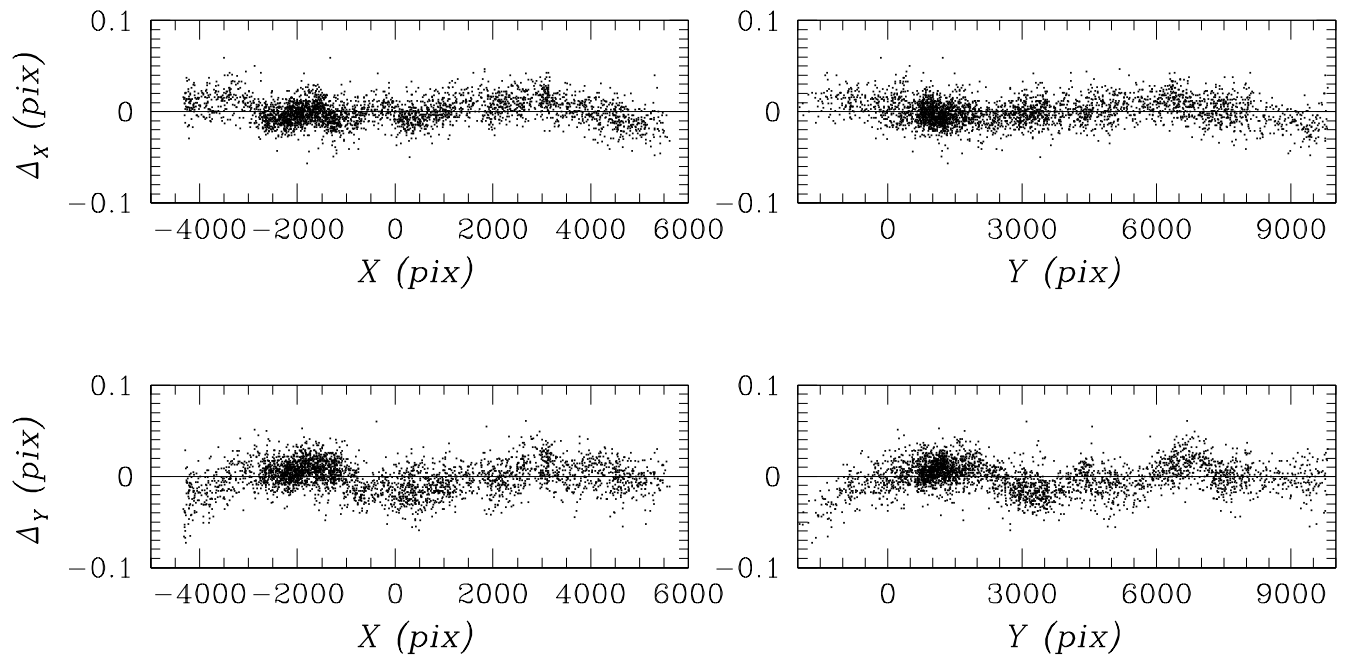

FIG. 5. - Positional residuals of stars in the overlap area observed by both, ACS/WFC and WFC3/UVIS. The global ACS/WFC pixel coordinates were transformed into the system of WFC3/UVIS global pixels. Small semi-periodic systematics with the amplitude up to $\sim 0.02$ WFC3/UVIS pixels are present. This appears to be the accuracy limit of our differential astrometry over scales of a few times the detector size. 

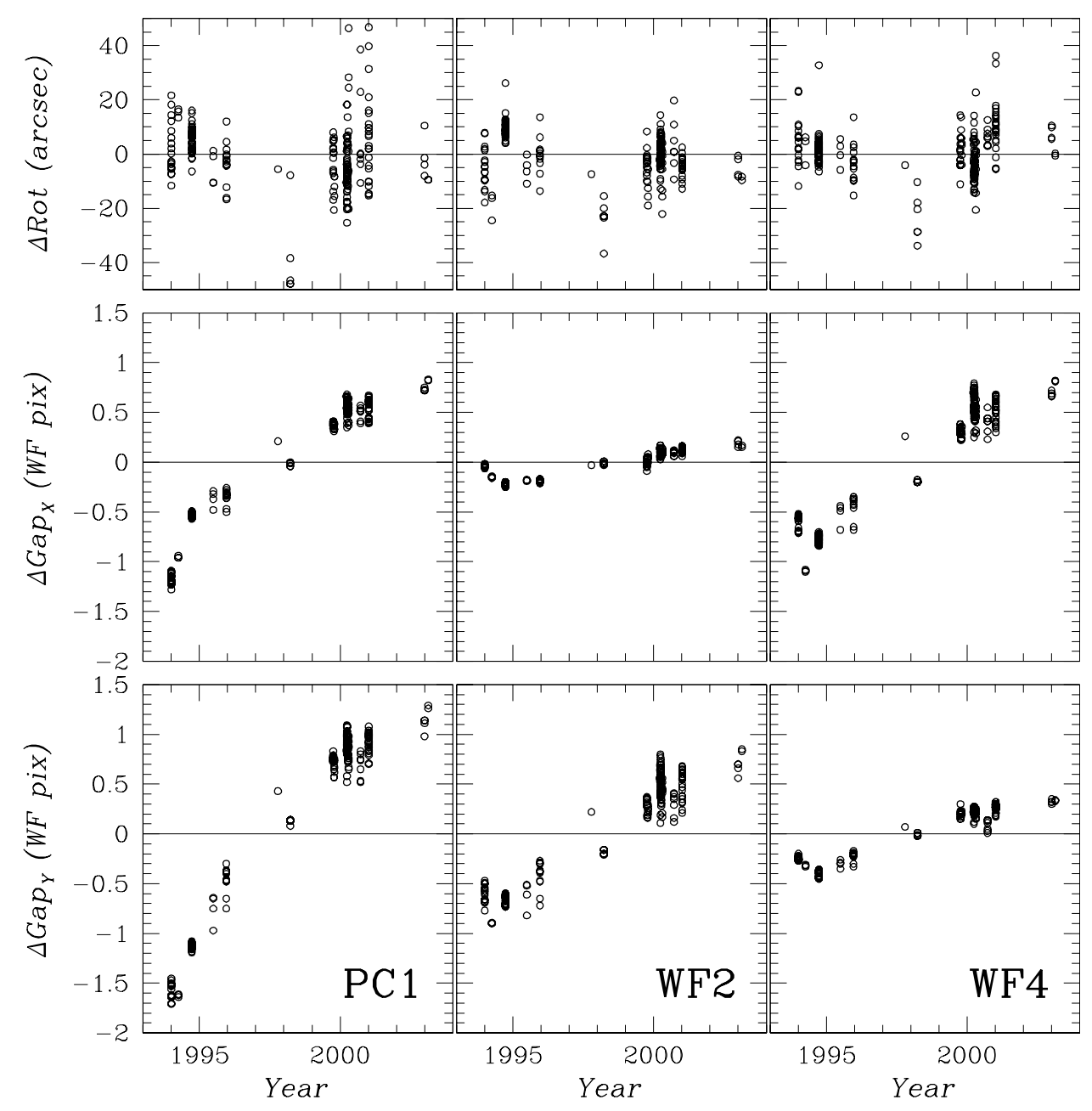

FIG. 6. - Variations over time in the chip rotation and inter-chip gap size. All chip parameters are relative to the WF3 chip of the WFPC2 camera. A zero in the ordinate corresponds to the mean chip parameters derived from a total of 219 WFPC2 frames, taken in the area of 30 Doradus. No distinction is made for the filter or exposure time, both of which may add noise to the datapoints. The observed trends or the lack of them are in excellent agreement with the earlier findings by Anderson \& King (2003). 

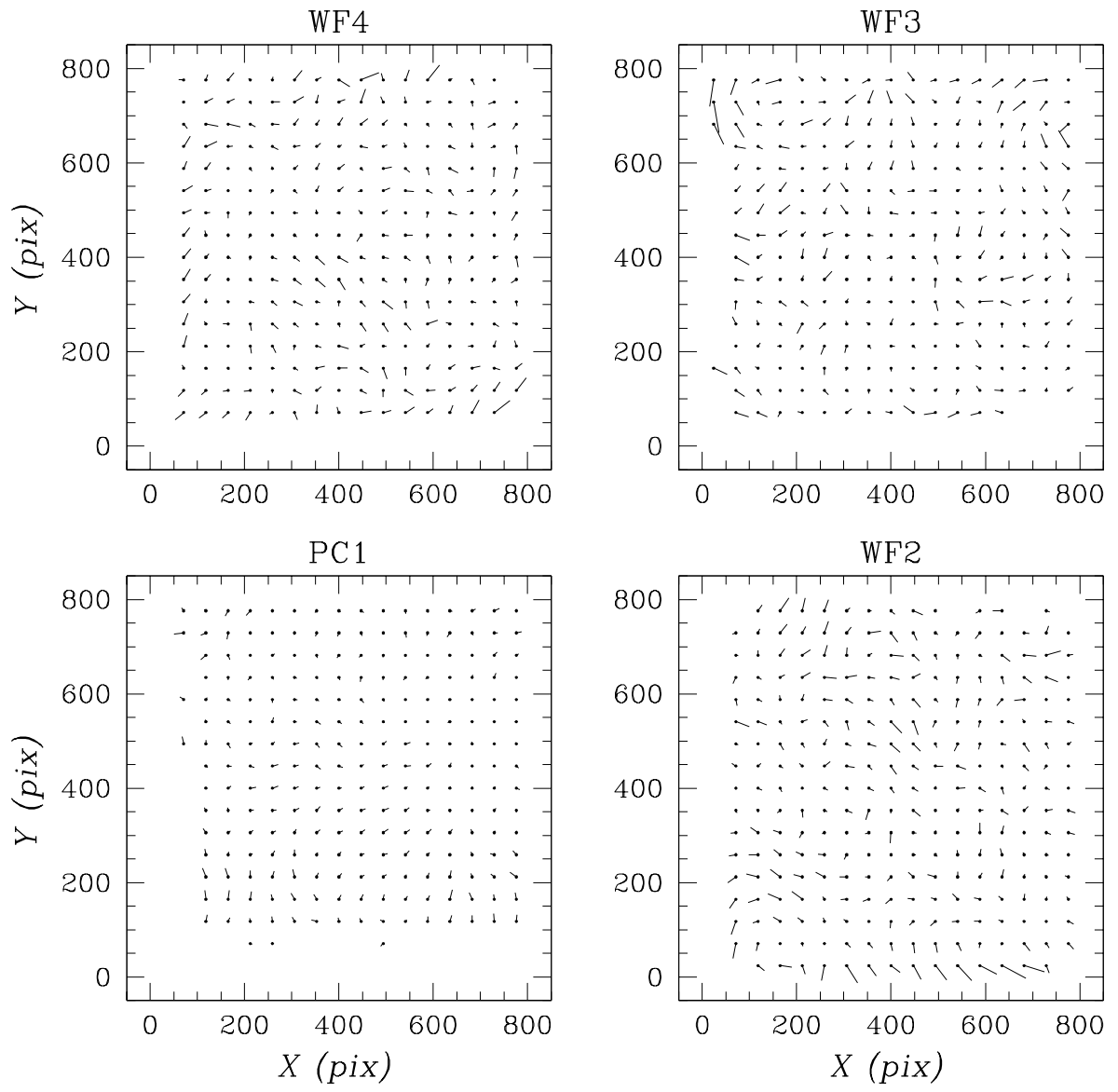

FIG. 7. - Two-dimensional maps of correlated residuals for WFPC2 chips. These smoothed residuals are on the pixel scale of the WFC3/UVIS camera and were applied to the distortion-corrected pixel coordinates of each WFPC2 chip. The size of each vectorial residual is magnified by a factor of 500. The maximum size of residuals in each coordinate ranges from $\sim 0.04$ pix on PC1 to $0.06-0.07$ pix on wide field (WF) CCD chips. These corrections can be used to enhance the astrometric precision of the standard distortion solution for WFPC2 available in Anderson \& King (2003). 


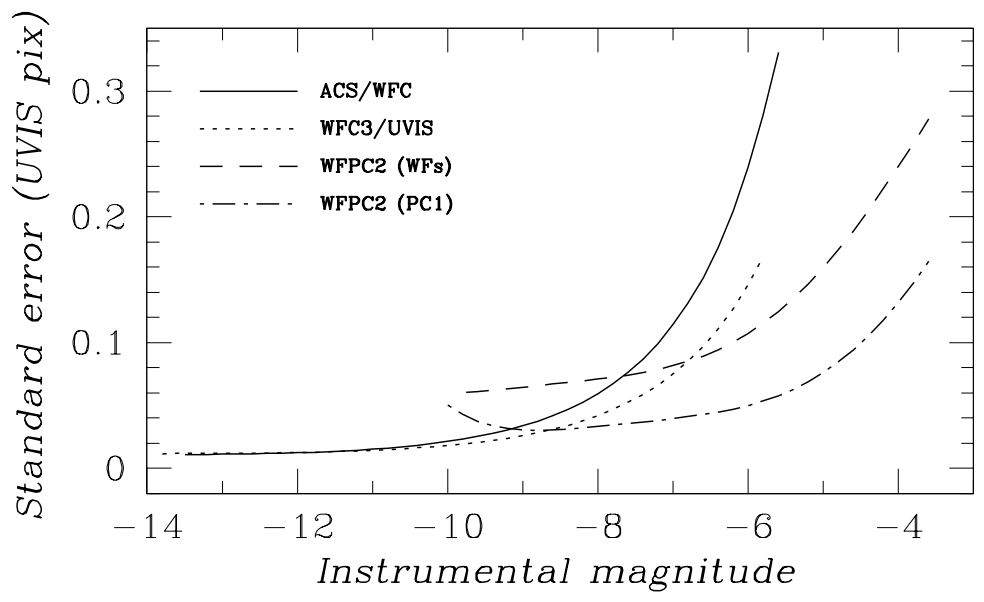

FIG. 8. - Expected standard centering error as a function of instrumental magnitude. The estimates for ACS/WFC and WFC3/UVIS are reproduced from Bellini et al. (2014). For WFPC2, we derived an error curve for PC1 and a single combined error curve for the wide-field WF2, WF3, and WF4 cameras. We note that the system of WFPC2 instrumental magnitudes on average is $~ 2.5$ mag fainter than that of $\mathrm{ACS} / \mathrm{WFC}$ and WFC3/UVIS. 


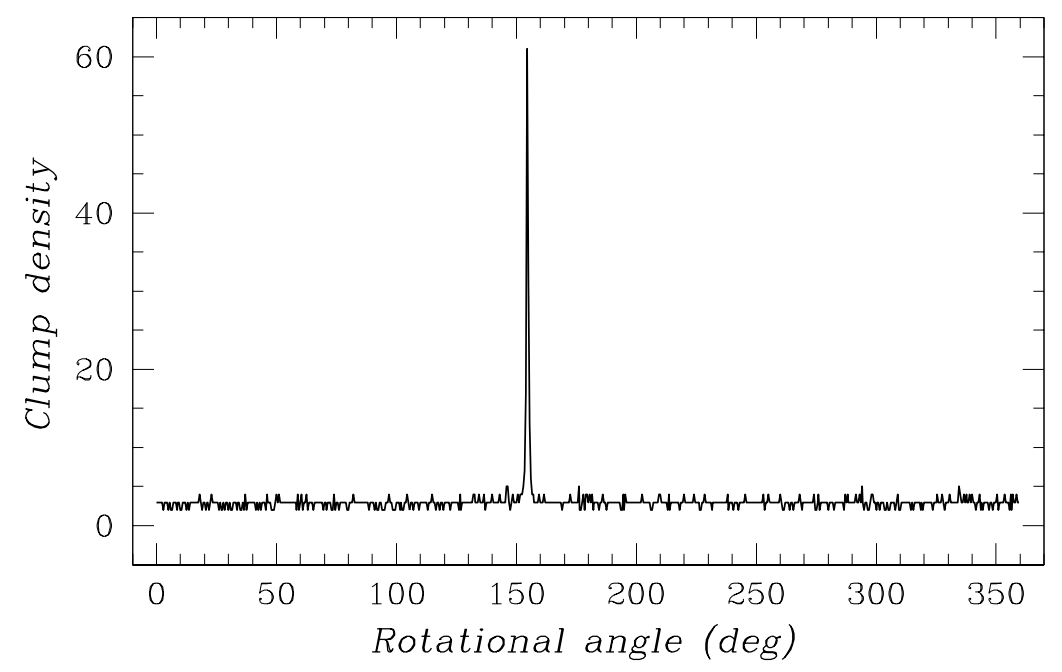

FIG. 9. - Example of finding a vectorial clump. All possible rotational angles between a target frame and the astrometric reference catalog were sampled. The position of a peak at $154^{\circ} .5$ indicates that angle (see Sect. 3.3.1). The full-width-half-maximum of the peak is only $\sim 1$. 9. 


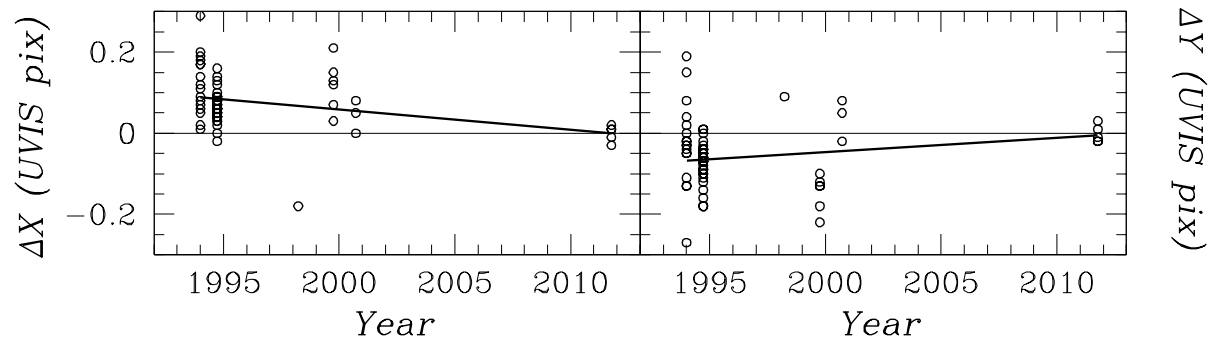

Fig. 10.- Weighted least-squares fit for a 17.9-mag star with a large number of measurements ( $n=68)$. Left panel: a fit of residuals in $X$ as a function of time; right panel: the same in $Y$-coordinate. All residuals are with respect to our astrometric reference frame. A detailed discussion is given in Sect. 3.3 .2 


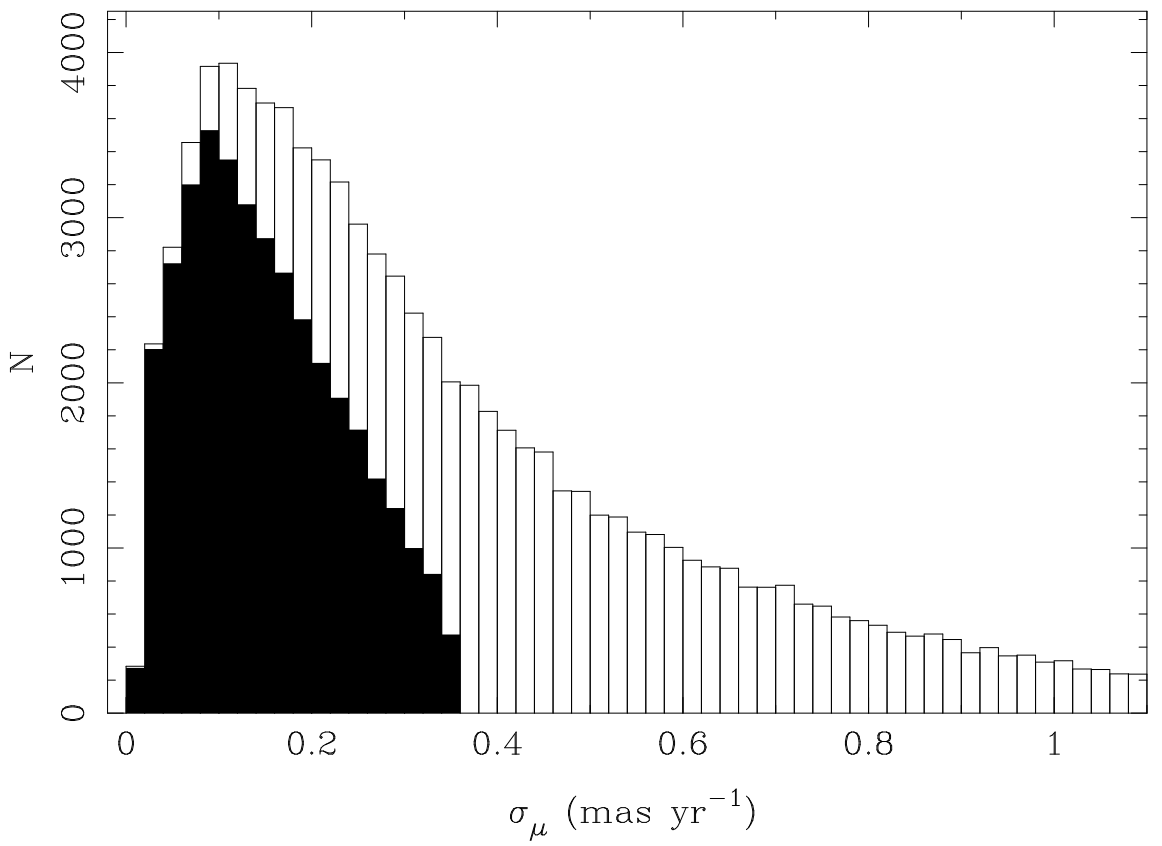

FIG. 11. - Histogram of proper-motion errors. White bins indicate the error distribution for all stars while the dark bins are limited to proper motions derived from more than 9 datapoints and cut at $\sigma_{\mu}<0.3$ mas $\mathrm{yr}^{-1}$. The distribution of errors is for the proper motions in one axis. The vast majority of stars with fewer datapoints are fainter than $m_{\mathrm{F} 775 \mathrm{~W}}=21$ and the formal high precision of their proper motions seen for some stars might be suspect due to the small number statistics. 


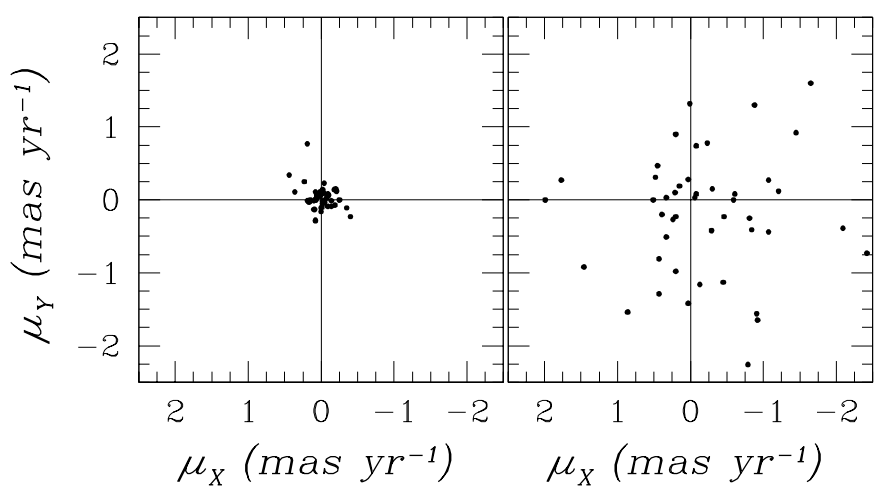

FIG. 12.- Vector-point diagram of selected VFTS stars. Left panel: a total of 50 VFTS stars in our proper motion catalog with a proper-motion error less that 0.13 mas $\mathrm{yr}^{-1}$. The proper-motion dispersion in either axis is 0.16 mas yr$^{-1}$. Right panel: the same stars but from Poleski et al. (2012). The dispersion of proper motions is at least 0.8-1.3 mas $\mathrm{yr}^{-1}$, depending upon the trimming of a few stars outside the shown limits. Our proper motions are clearly more precise and accurate. 


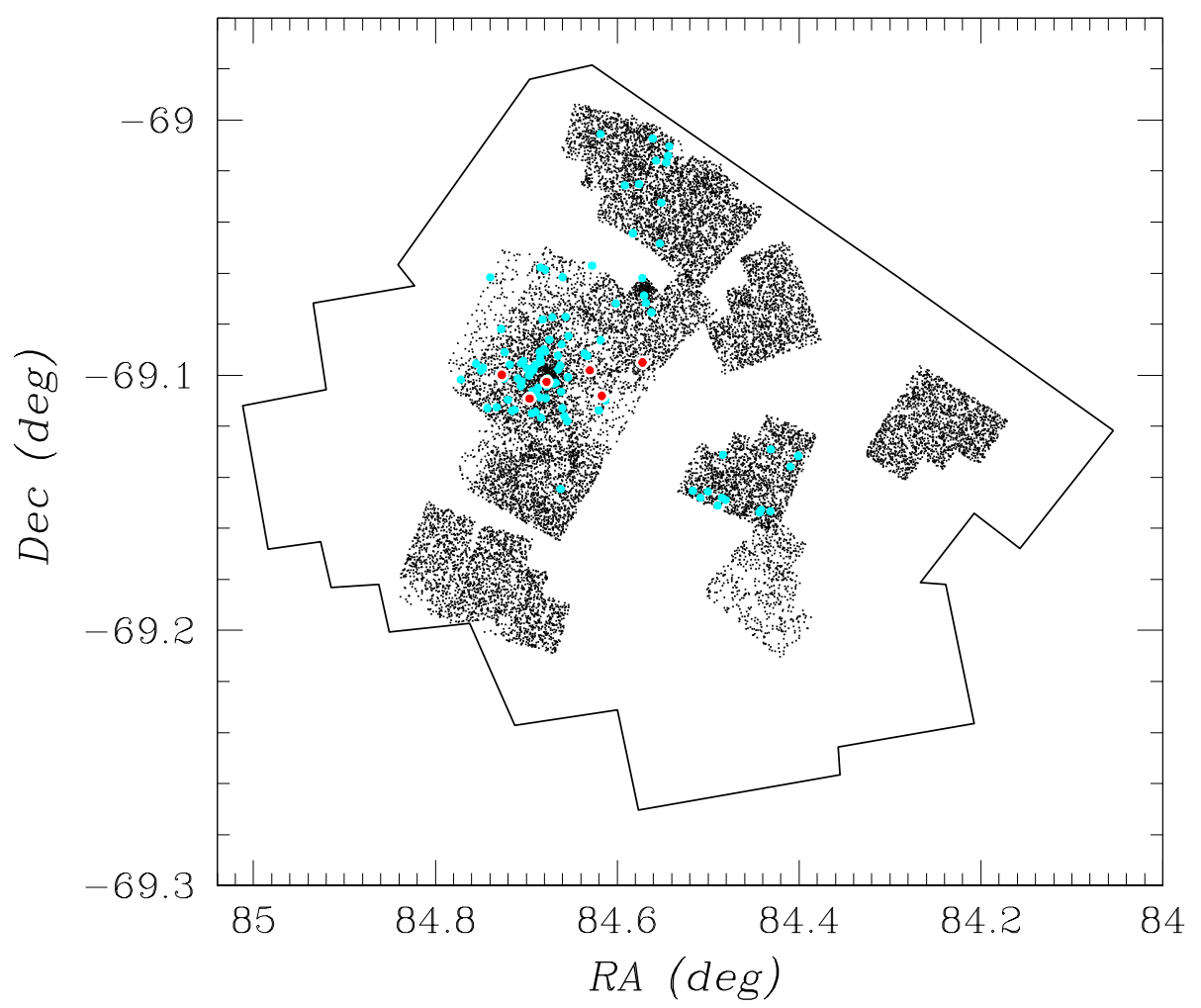

FIG. 13. - Distribution of stars with measured proper motions over the FOV. Only every 5-th star is plotted (black dots). The coverage pattern is defined by the availability of archival WFPC2 images. The rugged line has the same meaning as in Fig. 1 Large cyan dots indicate the location of 105 ostensibly faster-moving stars with proper motions exceeding $5 \sigma$ in at least one coordinate, where $\sigma$ is the actual proper-motion error of a star. Our best sample of possible OB runaway candidates is marked by large red dots. 


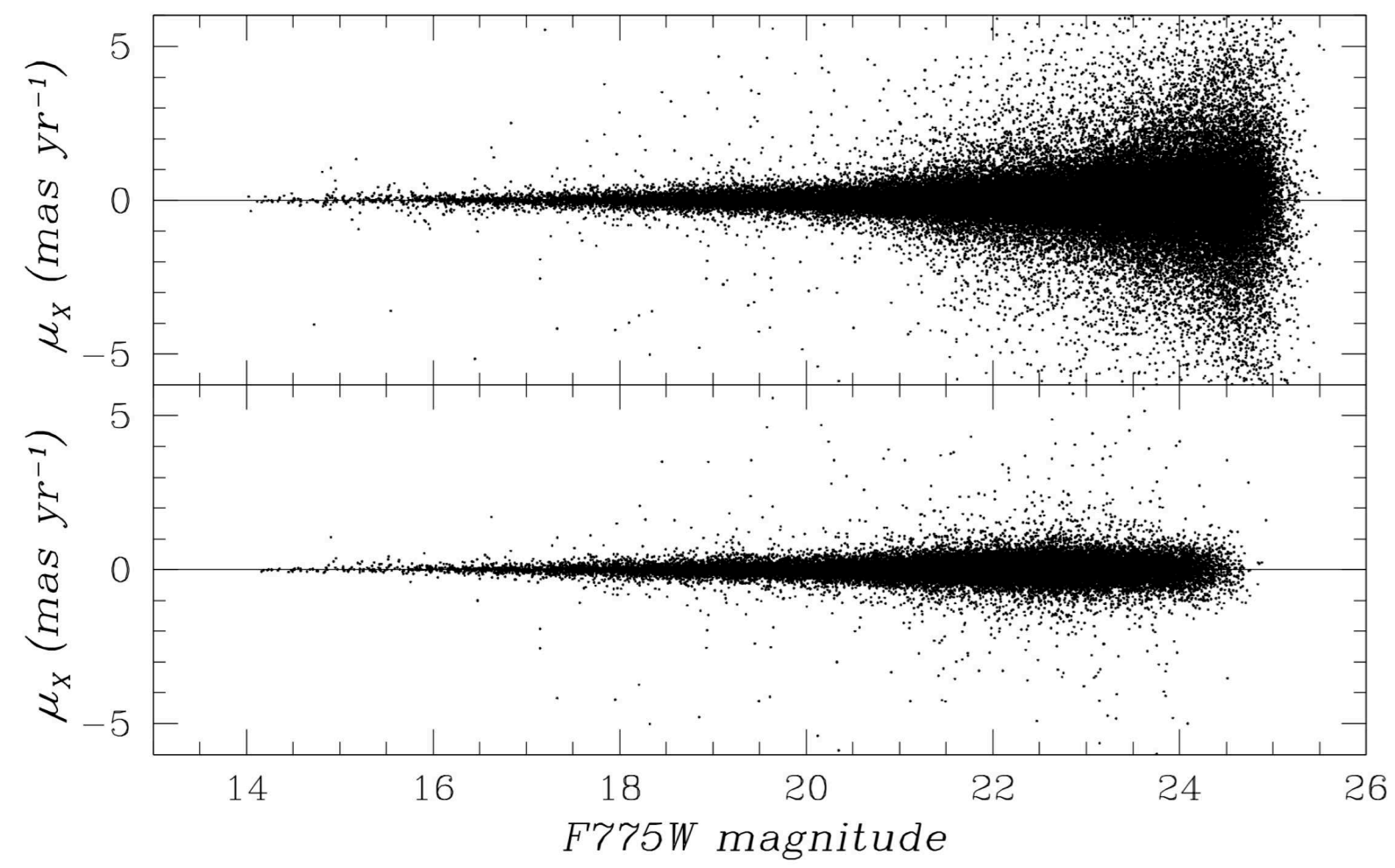

FIG. 14.- Relative proper motions in $X$ of stars in the 30 Dor region as a function of magnitude. The subscripts $X$ and $Y$ are a shorthand for RA $\cos (\mathrm{Dec})$ and Dec, and are used throughout the text, figures and tables. Upper panel: all 86,590 catalog stars are plotted. Lower panel: only stars with $\sigma_{\mu}<0.35$ mas $\mathrm{yr}^{-1}$ in both axes and with $n_{\text {frame }} \geq 9$ in the proper-motion fit are plotted. There are 37,026 such stars. The number of faint stars with unrealistic proper motions ( $\gtrsim 1$ mas $\left.\mathrm{yr}^{-1}\right)$ drops dramatically once the low-accuracy fits are deleted. 

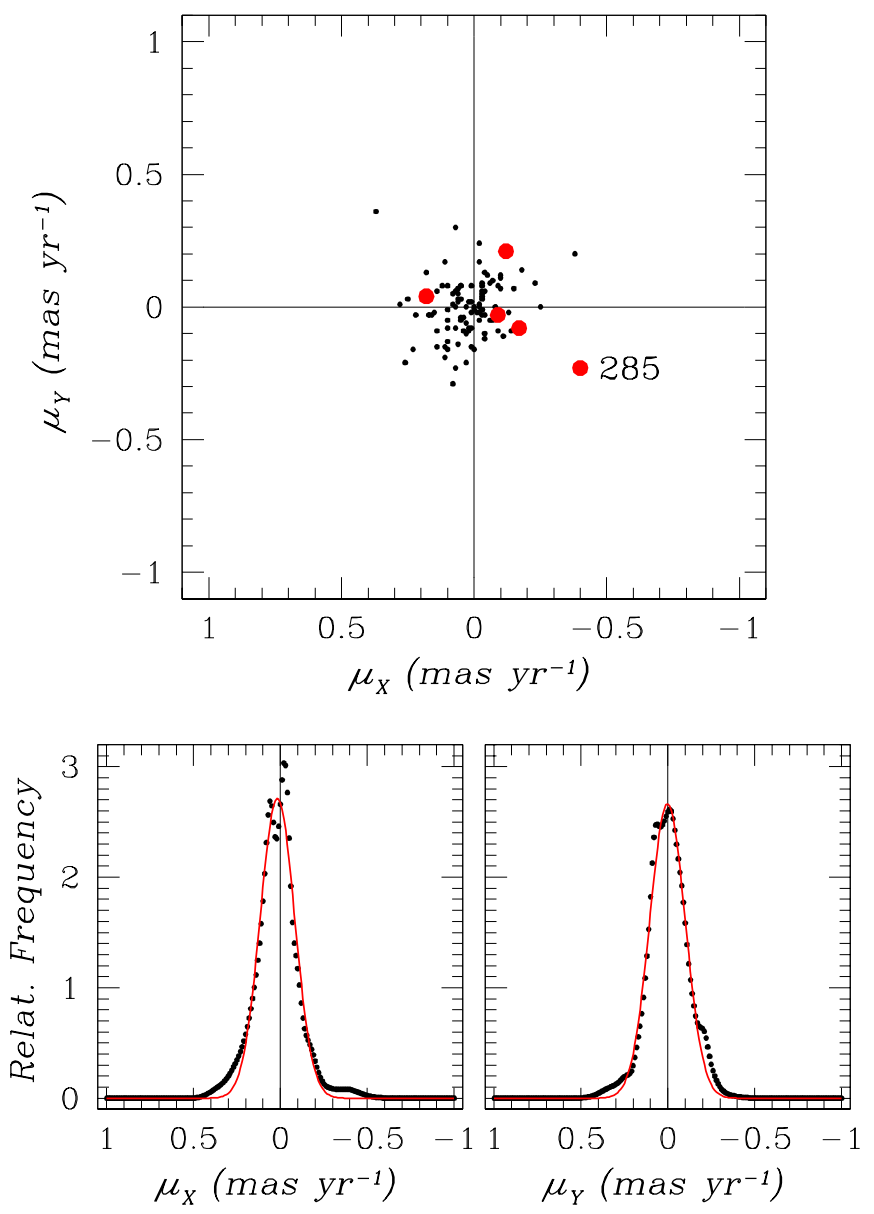

FIG. 15.- Distribution of well-measured proper motions for a total of 109 VFTS stars. Upper panel: a vector-point diagram. The LOS candidate runaway OB stars are highlighted by the red symbols. Star VFTS 285 is labeled because of its likely status of the LOS and a proper-motion runaway O star (see Sect. 4.2). Bottom two panels: a sum of unity Gaussians (black points) derived using the measured proper motions and their errors. Red curves represent a Gaussian fit to these distributions. In both coordinates the Gaussian width is only

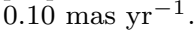




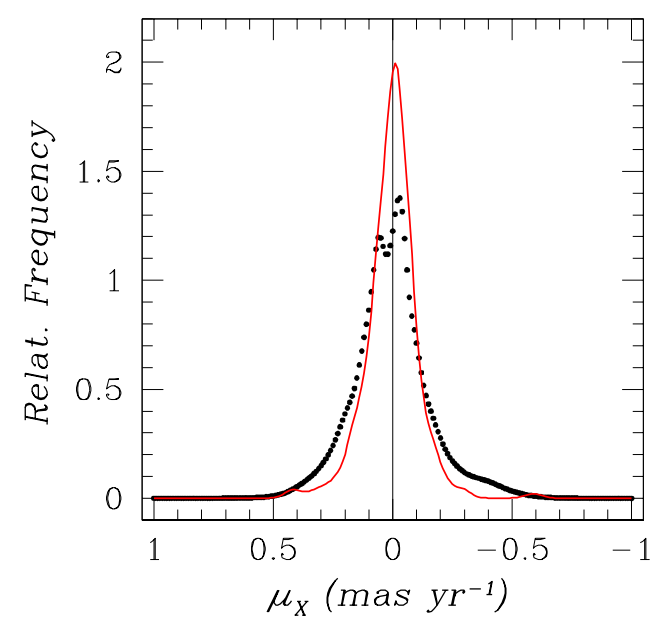

FIG. 16.- Distribution of proper motions for 199 VFTS stars. The black points show a superposition of unity Gaussians calculated from the measured proper motion in $X$. Similarly, the red curve is constructed by converting each offset-from-the-mean VFTS line-of-sight velocity into the equivalent of proper motion, convolved with the independently measured astrometric standard error. The distribution derived from the actual proper motions is somewhat broader than that derived from the LOS velocities. This indicates most likely that there are small systematic errors in the proper motion data that are comparable to the random errors. 

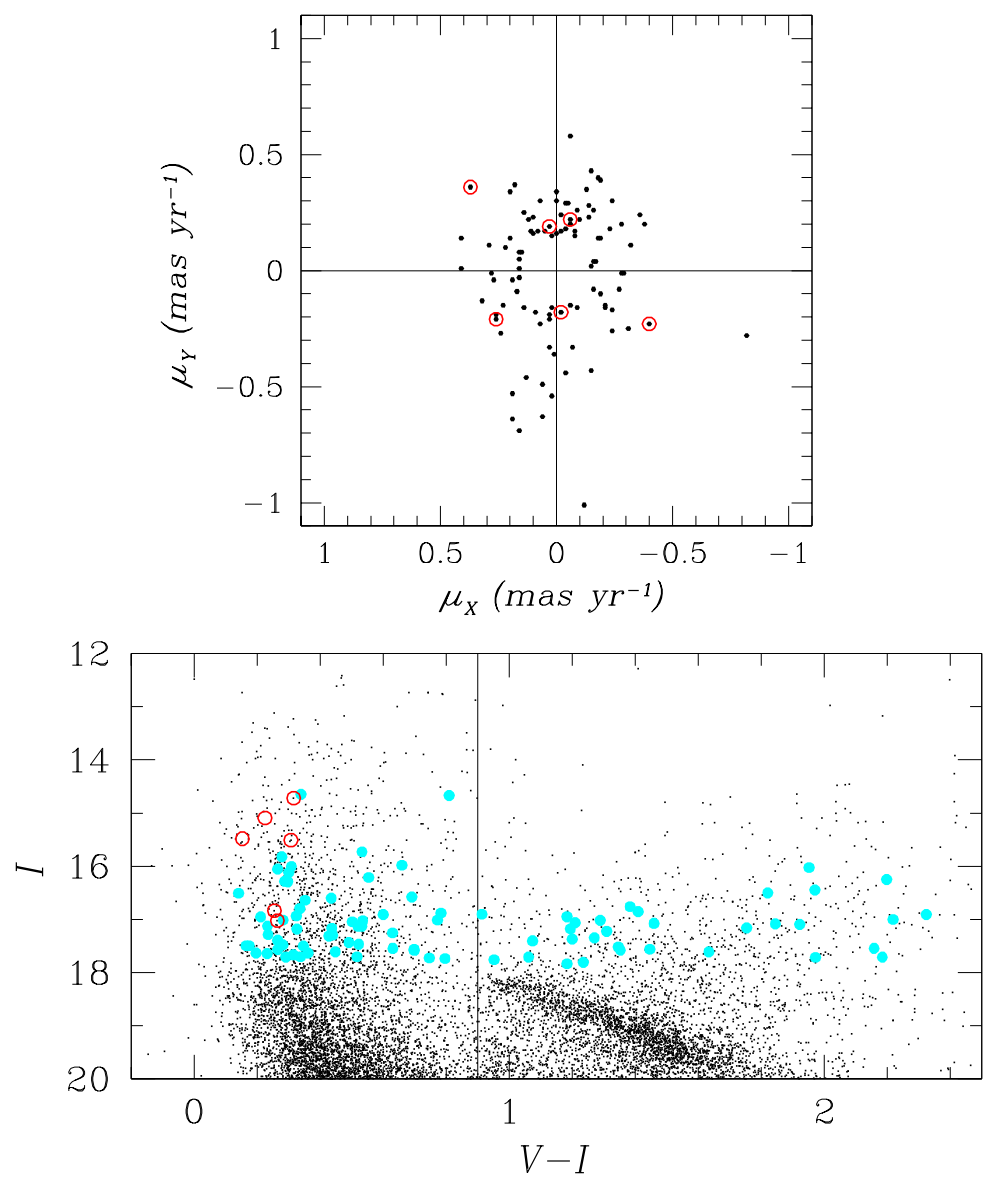

FIG. 17.- Possible fast-moving stars. Upper panel: a VPD of 105 bright stars $\left(m_{\mathrm{F} 775 \mathrm{~W}}<18\right)$ with statistically-significant proper motions at a $5 \sigma$ confidence. Red circles show our best six candidate runaway OB stars near R 136; bottom panel: a color-magnitude diagram indicating the possible fast-moving stars (large cyan points) and the candidate runaways (red circles). The black points represent a selection of stars with $I<20$ in the 30 Dor region. The vertical line at $V-I=0.9$ approximately separates the domain of OB stars from the brighter field RGB stars. 


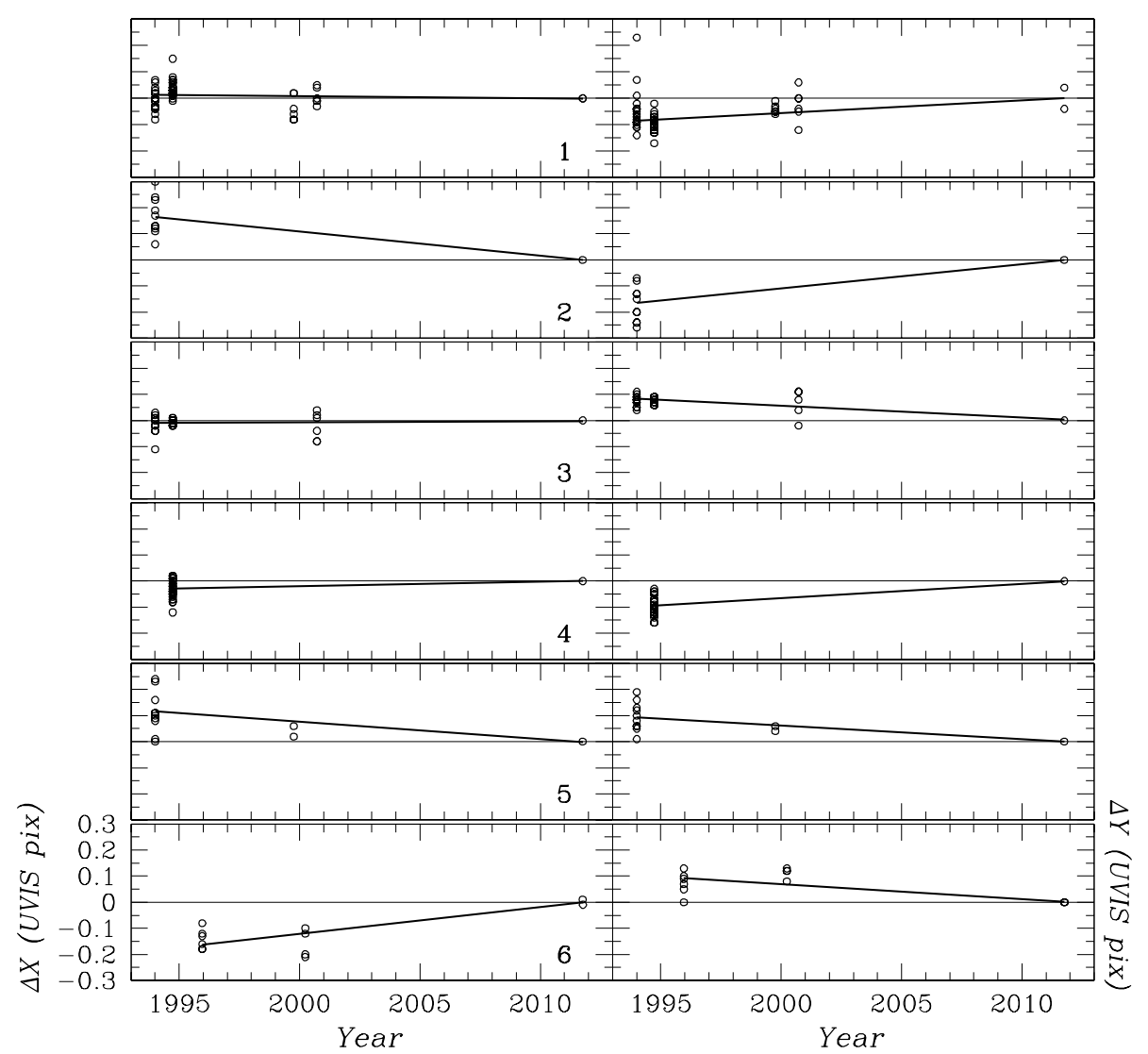

FIG. 18. - Weighted least-squares proper-motion fit for six candidate runaway OB stars. See Sect. 4.2 Fig. 10 and Table 3 for details. 


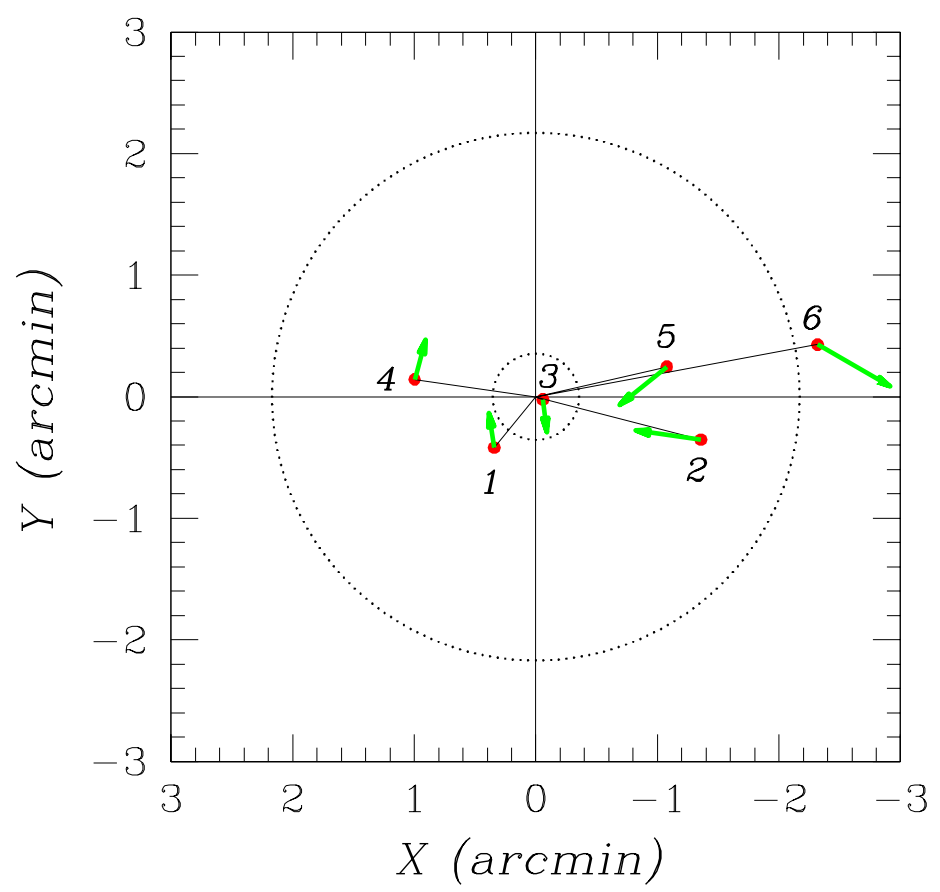

Fig. 19.- Location and proper-motion vector of six candidate runaway OB stars. The center of star cluster R 136 is at the zeropoint of gnomonic projection. To aid the eye, a line showing a possible path of ejection from R 136 is drawn for each star. Proper-motion vectors are shown by the green arrows. In this plot, $1^{\prime}$ corresponds to a proper motion of 1.5 mas $\mathrm{yr}^{-1}$. Out of six stars, only two have a direction of proper motion that suggests ejection from R 136. Dotted circles indicate the estimates of the half-light and the tidal radius. 
TABLE 1

WFPC2 OBSERVATIONS OF 30 DOR

\begin{tabular}{cccccc}
\hline \hline Program ID & $\mathrm{N}_{\text {obs }}$ & Filters & Epoch & RA (J2000) & Dec (J2000) \\
\hline 5114 & 48 & F555W,F814W & 1994.74 & $5: 38: 43$ & $-69: 06: 05$ \\
5584 & 4 & F656N,F675W & 1995.50 & $5: 37: 01$ & $-69: 07: 14$ \\
5589 & 21 & F336W,F555W,F814W,F547M,F502N,F656N,F673N & 1994.01 & $5: 38: 42$ & $-69: 06: 00$ \\
6122 & 13 & F555W,F814W,F502N,F656N,F673N & 1996.96 & $5: 38: 17$ & $-69: 04: 02$ \\
6251 & 1 & F814W & 1995.50 & $5: 37: 01$ & $-69: 07: 14$ \\
6540 & 8 & F656N & 1998.24 & $5: 38: 52$ & $-69: 08: 07$ \\
7786 & 1 & F606W & 1997.79 & $5: 37: 45$ & $-69: 10: 59$ \\
8059 & 4 & F606W,F814W & 1999.79 & $5: 39: 08$ & $-69: 10: 40$ \\
8059 & 4 & F606W,F814W & 2000.80 & $5: 38: 52$ & $-69: 11: 08$ \\
8059 & $44^{\mathrm{a}}$ & F606W,F814W & 2001.02 & $5: 38: 16$ & $-69: 01: 24$ \\
8059 & 23 & F606W,F814W,F656N & 2000.25 & $5: 38: 11$ & $-69: 08: 20$ \\
8090 & 8 & F606W & 2000.73 & $5: 38: 46$ & $-69: 05: 10$ \\
8163 & 8 & F502N,F656N,F673N & 2000.31 & $5: 38: 48$ & $-69: 04: 40$ \\
8163 & 8 & F502N,F656N,F673N & 1999.24 & $5: 38: 34$ & $-69: 06: 02$ \\
8163 & 8 & F555W,F814673N & 1999.76 & $5: 38: 44$ & $-69: 07: 44$ \\
8163 & 6 & F555W,F814W & 2000.22 & $5: 38: 02$ & $-69: 04: 33$ \\
8163 & 6 & F606W & 2001.02 & $5: 37: 49$ & $-69: 02: 14$ \\
8436 & 1 & F606W & 2003.00 & $5: 38: 44$ & $-69: 08: 27$ \\
8883 & 1 & F606W & & & \\
9676 & 6 & & & \\
& & & & & \\
\hline
\end{tabular}

a Mean coordinates given. The actual range in RA is from 5:38:08 to 5:38:24 and in Dec is from -69:01:03 to -69:01:46. 
TABLE 2

RMS SCATTER OF VARIOUS REFERENCE-FRAME SOLUTIONS.

\begin{tabular}{lcllcr}
\hline \hline Camera & Field type & rms in X (mas) & rms in Y (mas) & $\mathrm{N}_{\text {sol }}$ & $\mathrm{N}_{\text {stars }}$ \\
\hline ACS/WFC & frame-tile & $0.66(4)$ & $0.96(4)$ & 44 & 3200 \\
ACS/WFC & tile-strip & $0.58(8)$ & $0.65(7)$ & 13 & 2800 \\
ACS/WFC & strip-strip & 0.83 & 0.83 & 1 & 7903 \\
WFC3/UVIS & frame-tile & $0.60(4)$ & $0.74(5)$ & 44 & 1100 \\
WFC3/UVIS & tile-strip & $0.45(2)$ & $0.52(5)$ & 13 & 940 \\
WFC3/UVIS & strip-strip & 0.60 & 0.88 & 1 & 2596 \\
ACS-WFC3 & strip-strip & 0.56 & 0.68 & 1 & 3322 \\
& & & & & \\
\hline
\end{tabular}


Table 3. Candidate OB Proper-motion Runaway stars (SEe Section 4.2 for units and details)

\begin{tabular}{|c|c|c|c|c|c|c|c|c|c|c|c|c|c|c|}
\hline ID & VFTS & $m_{\mathrm{F} 775 \mathrm{~W}}$ & $m_{\mathrm{F} 555 \mathrm{~W}}-m_{\mathrm{F} 775 \mathrm{~W}}$ & $R A$ (deg) & Dec (deg) & $\mu_{X}$ & $\mu_{Y}$ & $\sigma_{\mu_{X}}$ & $\sigma_{\mu_{Y}}$ & $\chi_{X}^{2}$ & $\chi_{Y}^{2}$ & $Q_{X}$ & $Q_{Y}$ & $\mathrm{~N}_{\text {frame }}$ \\
\hline 1 & $\cdots$ & 16.836 & 0.255 & 84.6963497 & -69.1091730 & 0.03 & 0.19 & 0.02 & 0.03 & 0.32 & 0.91 & 1.00 & 0.67 & 62 \\
\hline 2 & 350 & 14.718 & 0.316 & 84.6169242 & -69.1080834 & 0.37 & 0.36 & 0.05 & 0.06 & 1.03 & 1.07 & 0.41 & 0.38 & 11 \\
\hline 3 & $\cdots$ & 15.508 & 0.308 & 84.6776254 & -69.1025824 & -0.02 & -0.18 & 0.02 & 0.02 & 0.32 & 0.22 & 1.00 & 1.00 & 42 \\
\hline 4 & $\cdots$ & 17.024 & 0.264 & 84.7270015 & -69.0998315 & -0.06 & 0.22 & 0.02 & 0.02 & 0.23 & 0.23 & 1.00 & 1.00 & 31 \\
\hline 5 & 373 & 15.096 & 0.225 & 84.6301239 & -69.0980782 & 0.26 & -0.21 & 0.06 & 0.04 & 1.29 & 0.49 & 0.22 & 0.91 & 13 \\
\hline 6 & 285 & 15.482 & 0.153 & 84.5722167 & -69.0950254 & -0.40 & -0.23 & 0.06 & 0.05 & 0.79 & 0.56 & 0.64 & 0.85 & 12 \\
\hline
\end{tabular}

